Supporting Information

for

\title{
Switchable Ring-Contractive Extrusion Reactions of 2,5-Dihydro-1,4,5-thiadiazepine S-Oxides: Entries to Pyridazines or Pyrazoles
}

Bin Cheng, ${ }^{*} \dagger, \star$ Y Yntong Li, ${ }^{\ddagger}, \perp$ Hui Li, ${ }^{\ddagger}, \perp$ Xinping Zhang, ${ }^{\ddagger}$ Taimin Wang, ${ }^{\dagger}$ Yun $\mathrm{Li},{ }^{\ddagger}$ and Hongbin Zhai ${ }^{*} \dagger, \ddagger, \S$

${ }^{\dagger}$ Institute of Marine Biomedicine, Shenzhen Polytechnic, Shenzhen 518055, China

$¥$ State Key Laboratory of Applied Organic Chemistry, College of Chemistry and Chemical Engineering, Lanzhou University, Lanzhou 730000, China

${ }^{\S}$ State Key Laboratory of Chemical Oncogenomics, Shenzhen Engineering Laboratory of Nano Drug Slow-Release, Peking University Shenzhen Graduate School, Shenzhen 518055, China

Table of Contents

1. NMR spectra S2-S19

2. Crystal Structure Information of Compounds $2 \mathrm{a}$ and $3 \mathrm{~g}$ S20-S23 


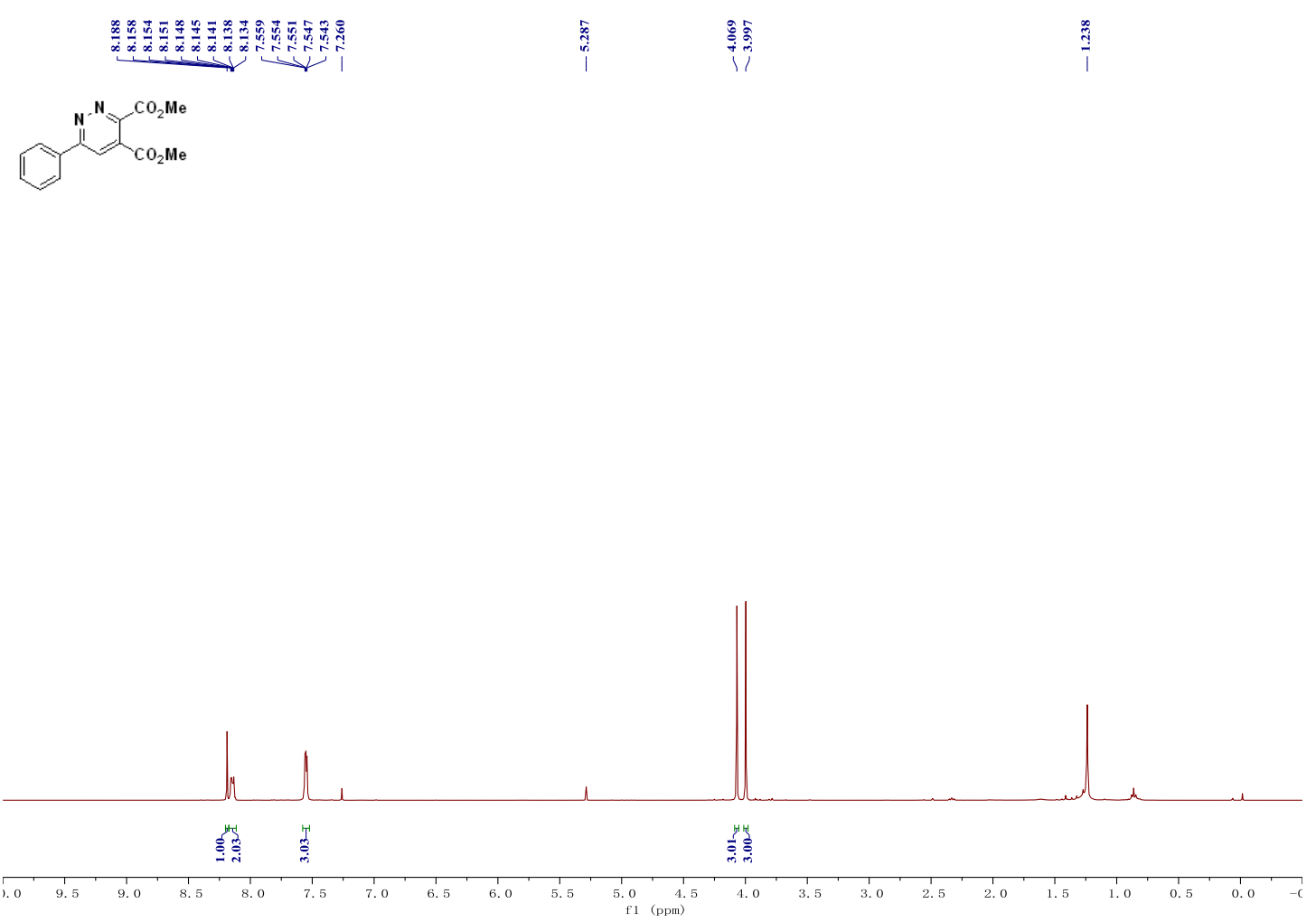

Fig. S1 ${ }^{1} \mathrm{H}$ NMR of compound $\mathbf{2 a}\left(400 \mathrm{MHz}, \mathrm{CDCl}_{3}\right)$.
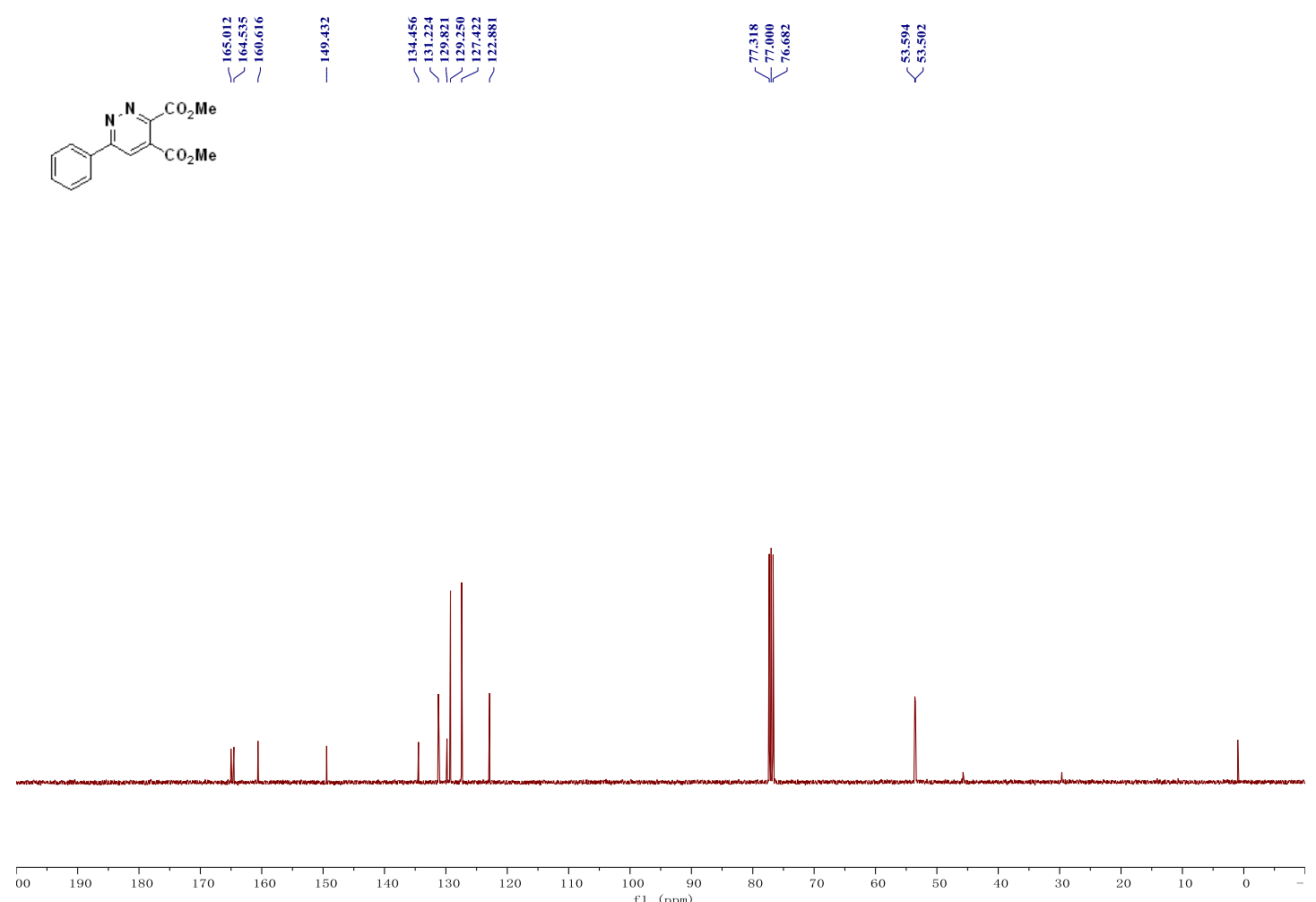

Fig. $\mathbf{S 2}{ }^{13} \mathrm{C}$ NMR of compound $\mathbf{2 a}\left(100 \mathrm{MHz}, \mathrm{CDCl}_{3}\right)$. 
MeO

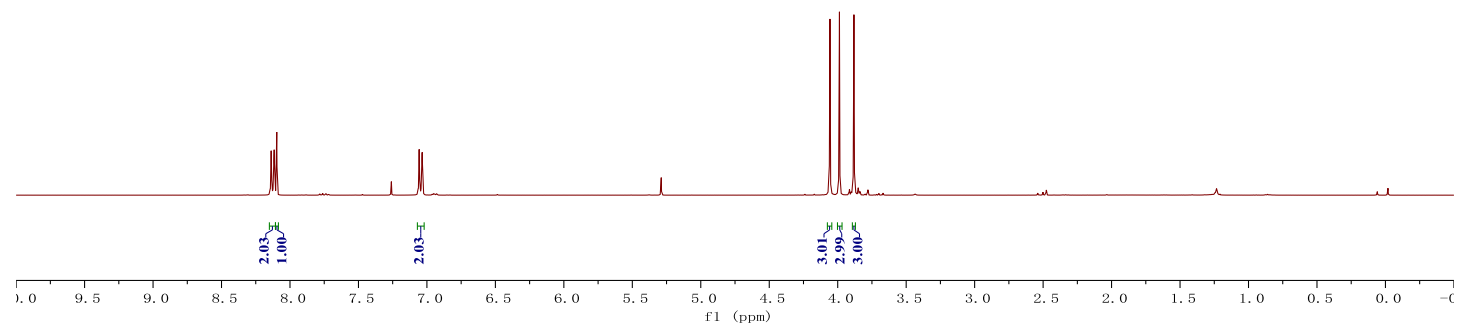

Fig. $\mathbf{S 3}{ }^{1} \mathrm{H}$ NMR of compound $\mathbf{2 b}\left(400 \mathrm{MHz}, \mathrm{CDCl}_{3}\right)$.
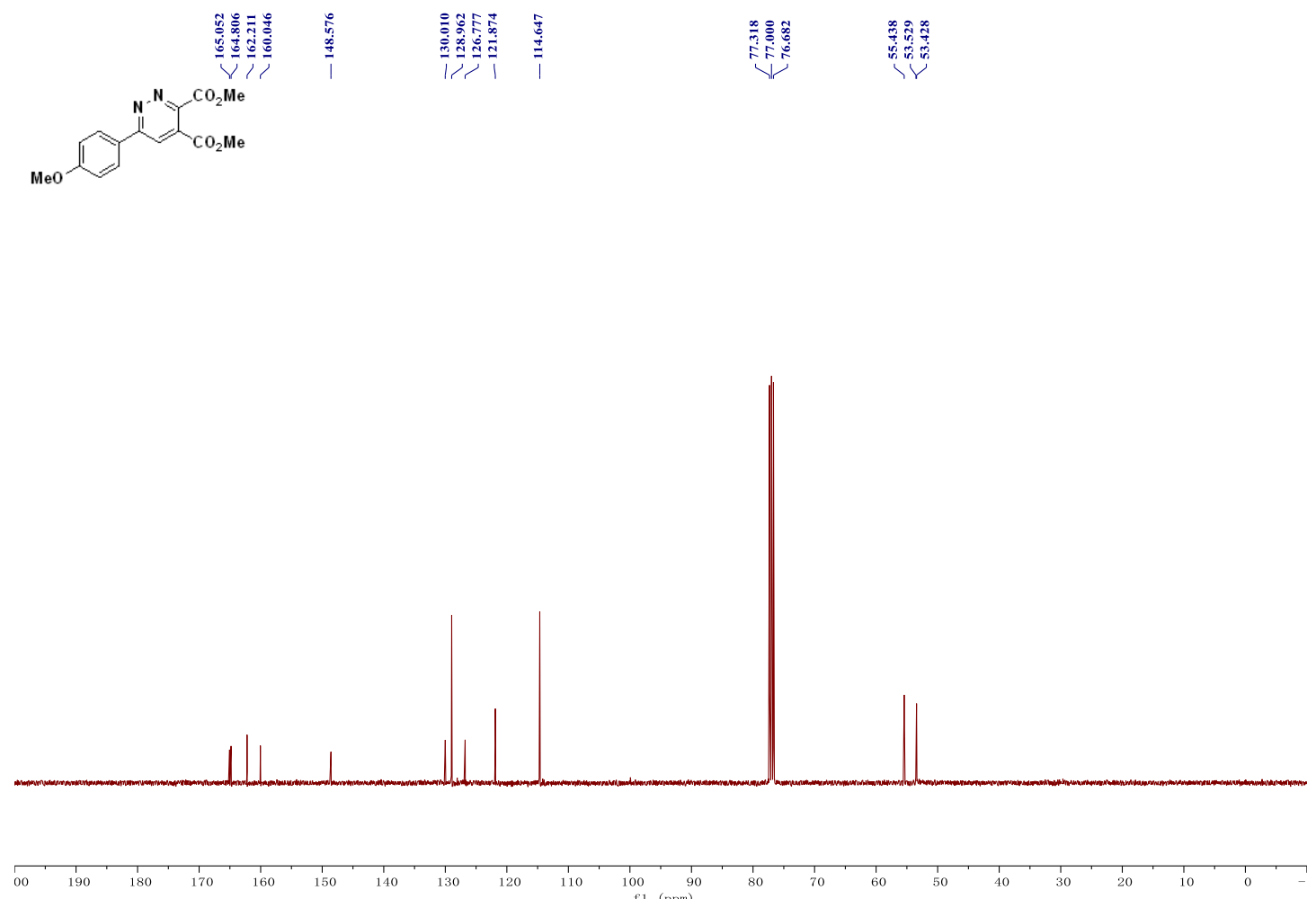

Fig. $\mathbf{S 4}{ }^{13} \mathrm{C}$ NMR of compound $\mathbf{2 b}\left(100 \mathrm{MHz}, \mathrm{CDCl}_{3}\right)$. 

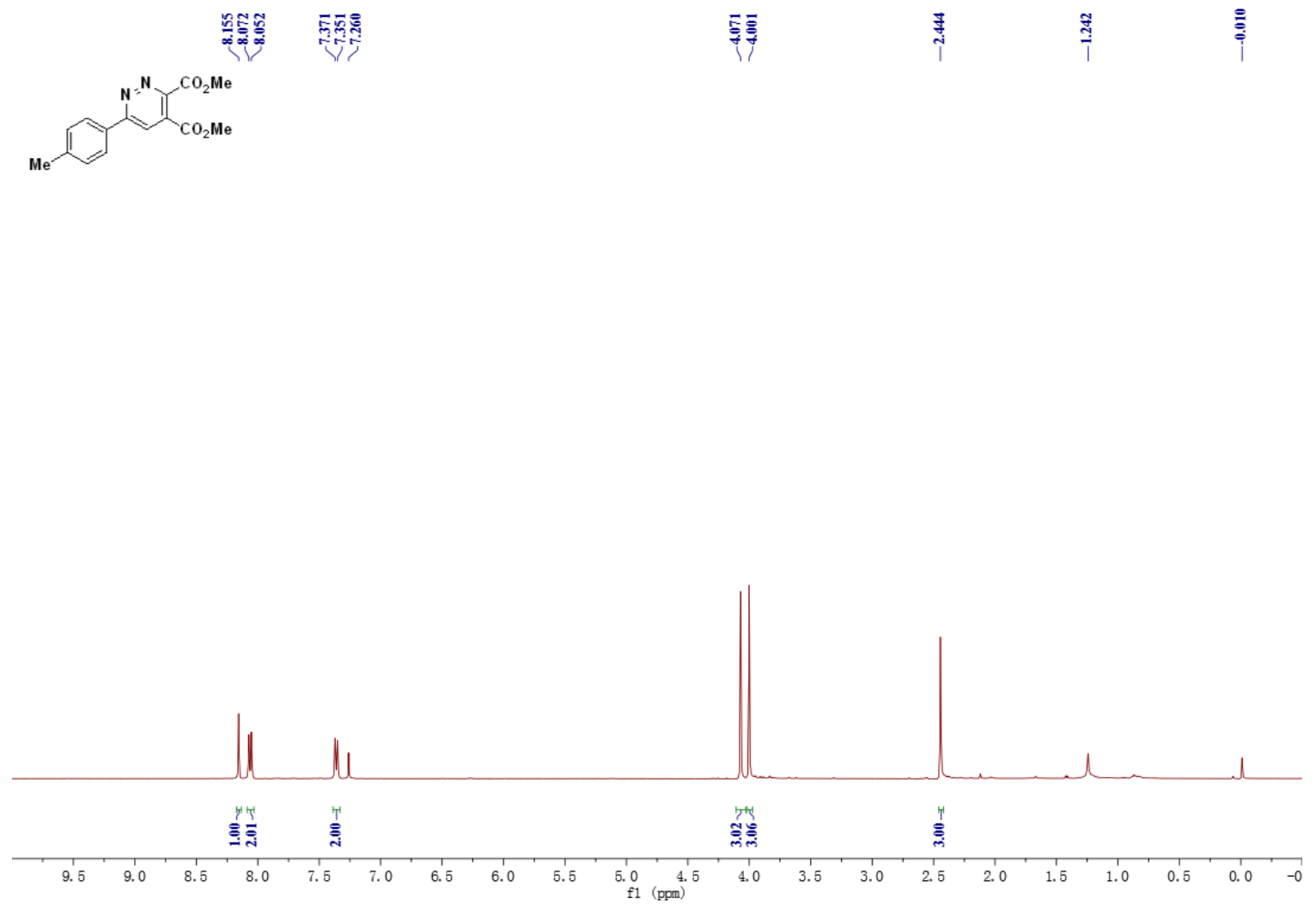

Fig. $\mathbf{S 5}{ }^{1} \mathrm{H}$ NMR of compound $2 c\left(400 \mathrm{MHz}, \mathrm{CDCl}_{3}\right)$.
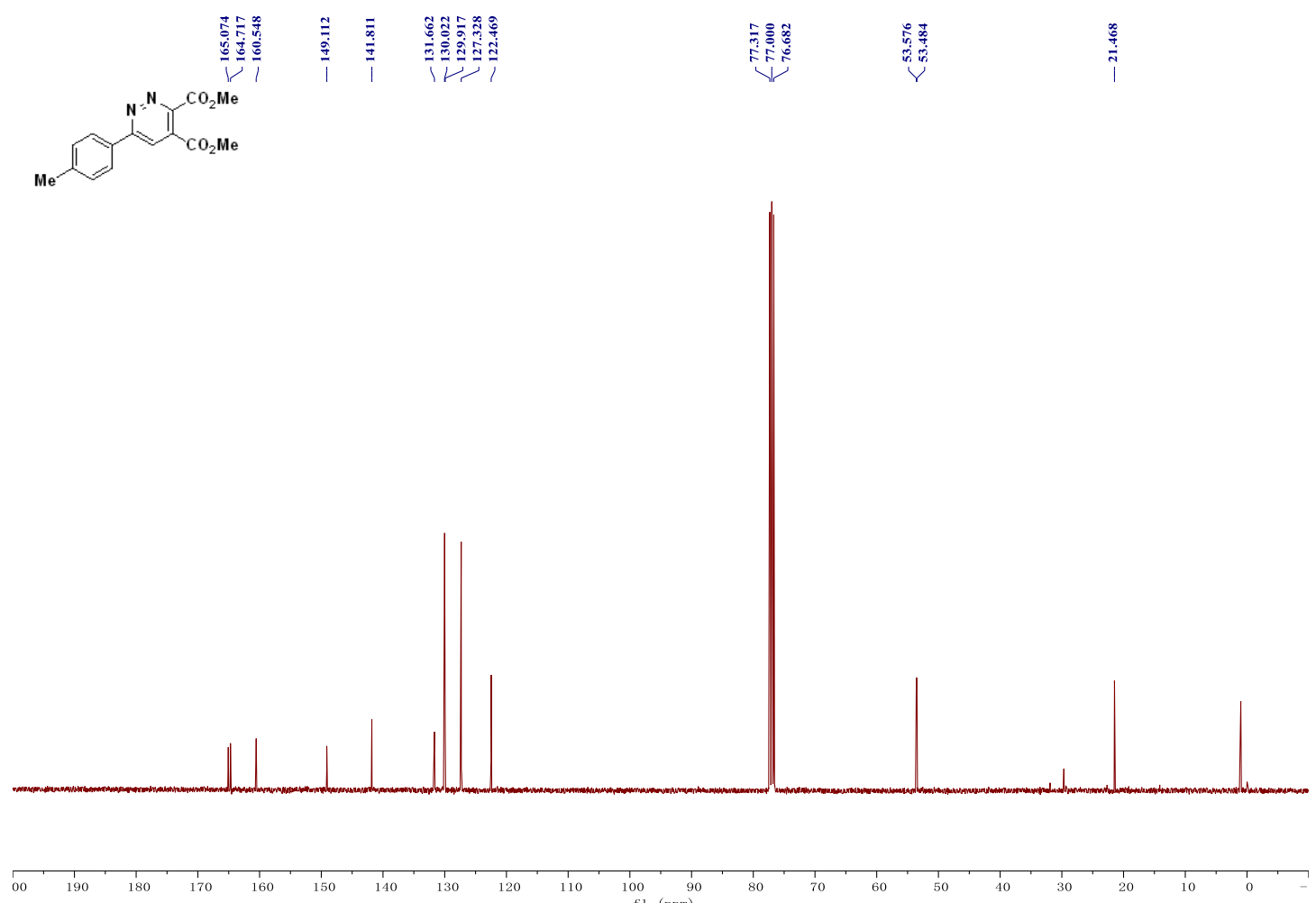

Fig. S6 ${ }^{13} \mathrm{C}$ NMR of compound $2 \mathrm{c}\left(100 \mathrm{MHz}, \mathrm{CDCl}_{3}\right)$. 

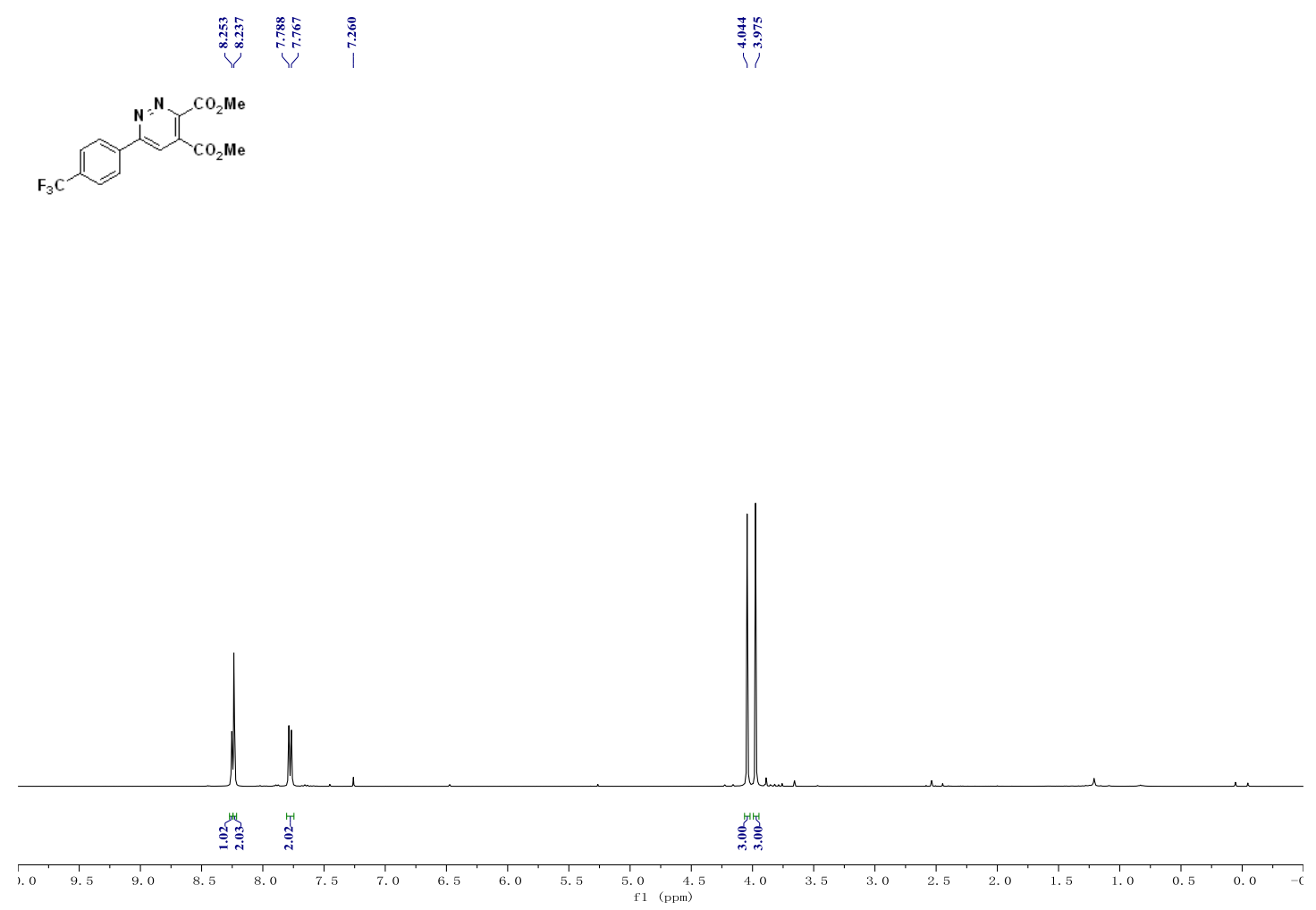

Fig. $\mathbf{S} 7{ }^{1} \mathrm{H}$ NMR of compound $2 \mathrm{~d}\left(400 \mathrm{MHz}, \mathrm{CDCl}_{3}\right)$.
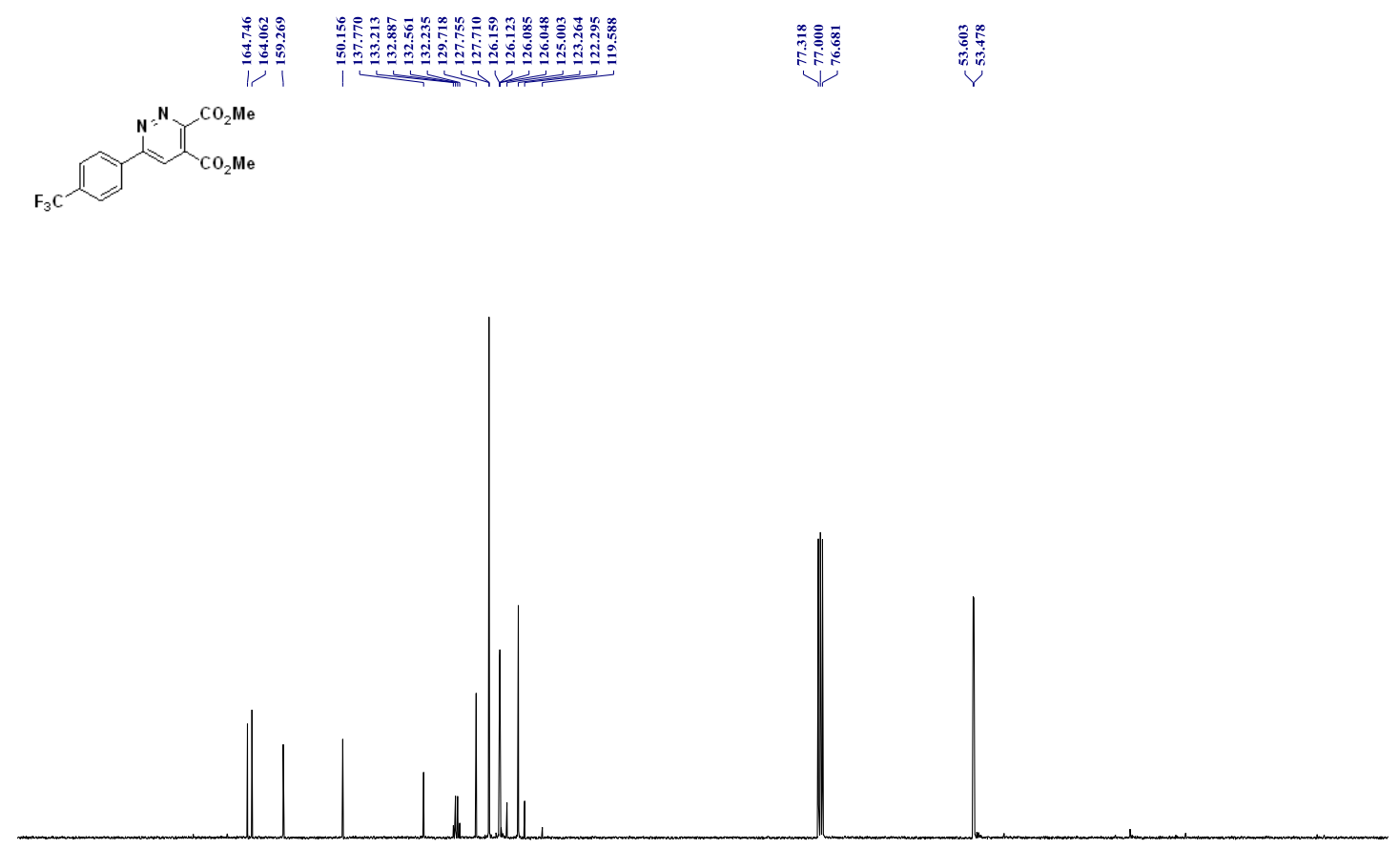

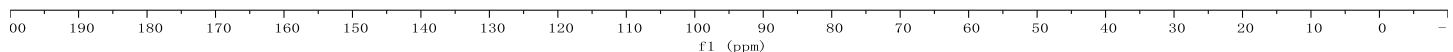

Fig. S8 ${ }^{13} \mathrm{C}$ NMR of compound $2 d\left(100 \mathrm{MHz}, \mathrm{CDCl}_{3}\right)$. 

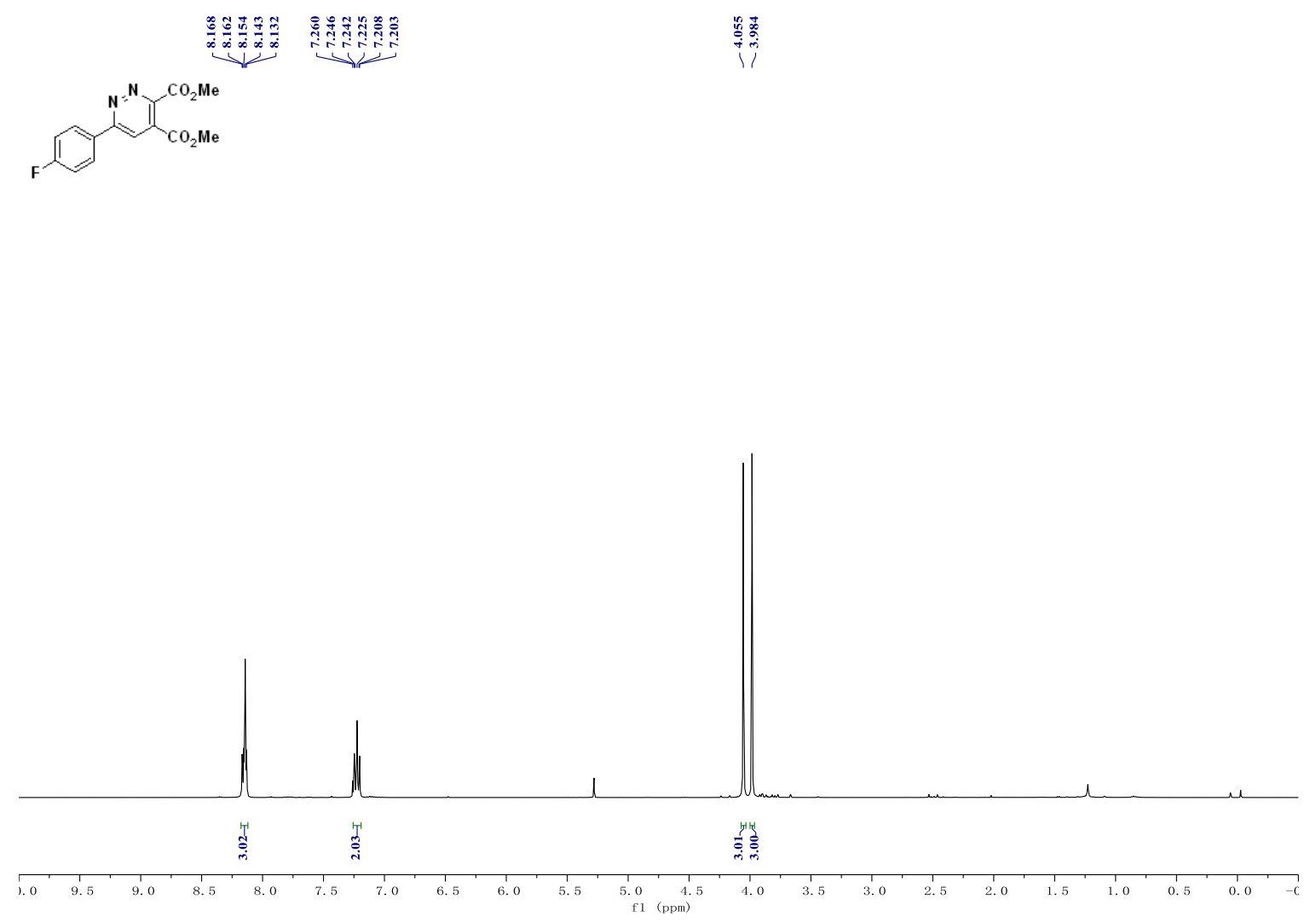

Fig. S9 ${ }^{1} \mathrm{H}$ NMR of compound $2 \mathrm{e}\left(400 \mathrm{MHz}, \mathrm{CDCl}_{3}\right)$.
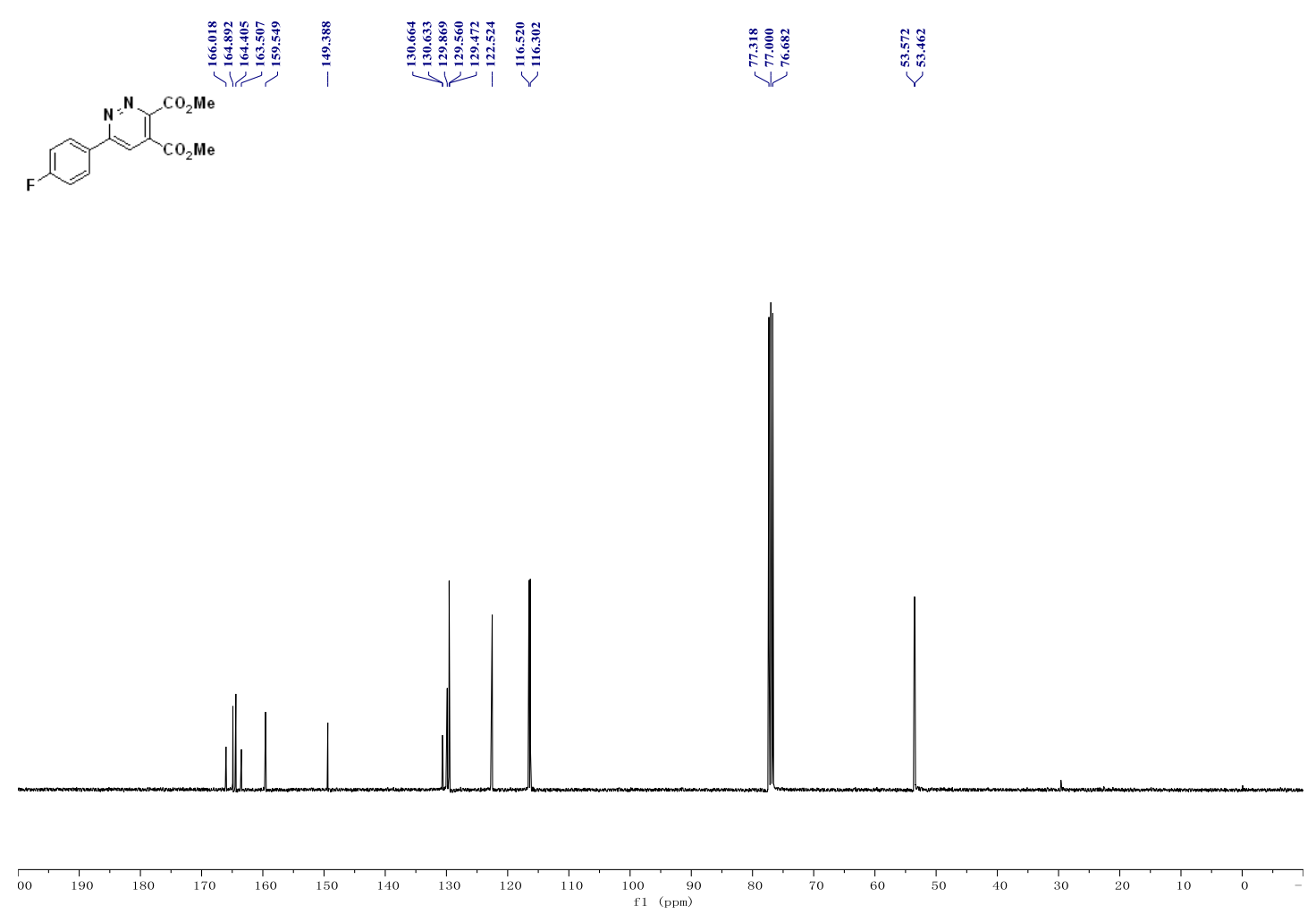

Fig. $\mathbf{S 1 0}{ }^{13} \mathrm{C}$ NMR of compound $2 \mathrm{e}\left(100 \mathrm{MHz}, \mathrm{CDCl}_{3}\right)$. 
$\overbrace{0}^{\mathrm{N}} \overbrace{\mathrm{CO}_{2} \mathrm{Me}}^{\mathrm{CO}_{2} \mathrm{Me}}$

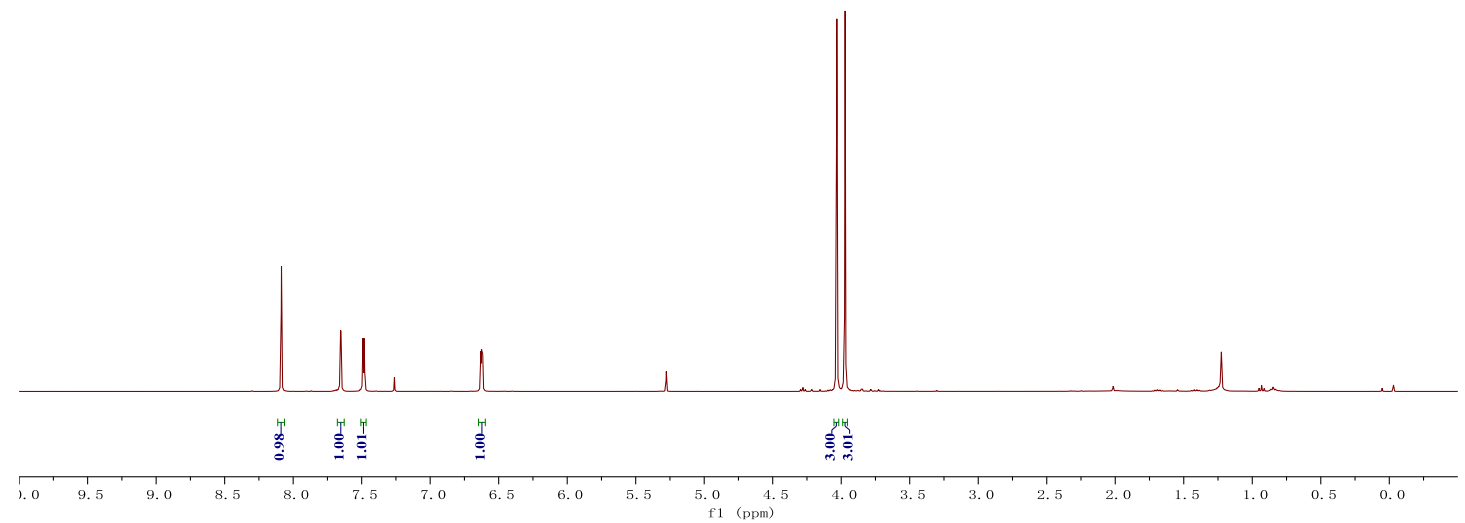

Fig. S11 ${ }^{1} \mathrm{H}$ NMR of compound $2 \mathbf{f}\left(400 \mathrm{MHz}, \mathrm{CDCl}_{3}\right)$.

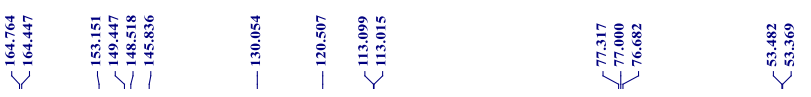

$$
\overbrace{0}^{-\mathrm{N}} \mathrm{CO}_{2}^{\mathrm{Me}}
$$
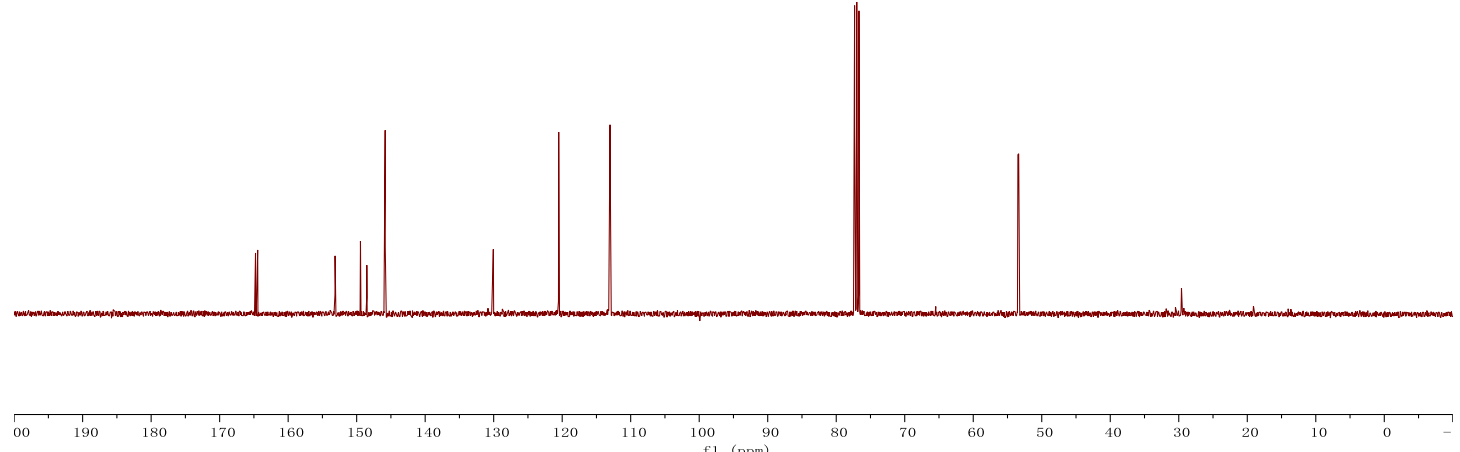

Fig. $\mathbf{S 1 2}{ }^{13} \mathrm{C}$ NMR of compound $2 \mathbf{f}\left(100 \mathrm{MHz}, \mathrm{CDCl}_{3}\right)$. 


$$
+\mathrm{CO}_{2} \mathrm{Me}
$$

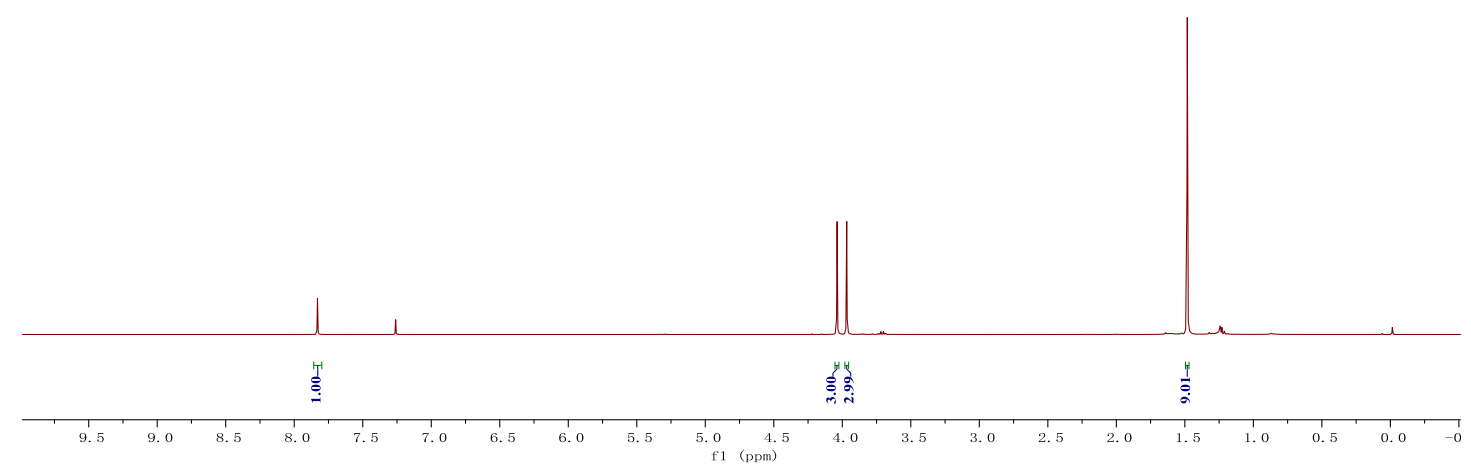

Fig. S13 ${ }^{1} \mathrm{H}$ NMR of compound 2 g (400 $\left.\mathrm{MHz}, \mathrm{CDCl}_{3}\right)$.
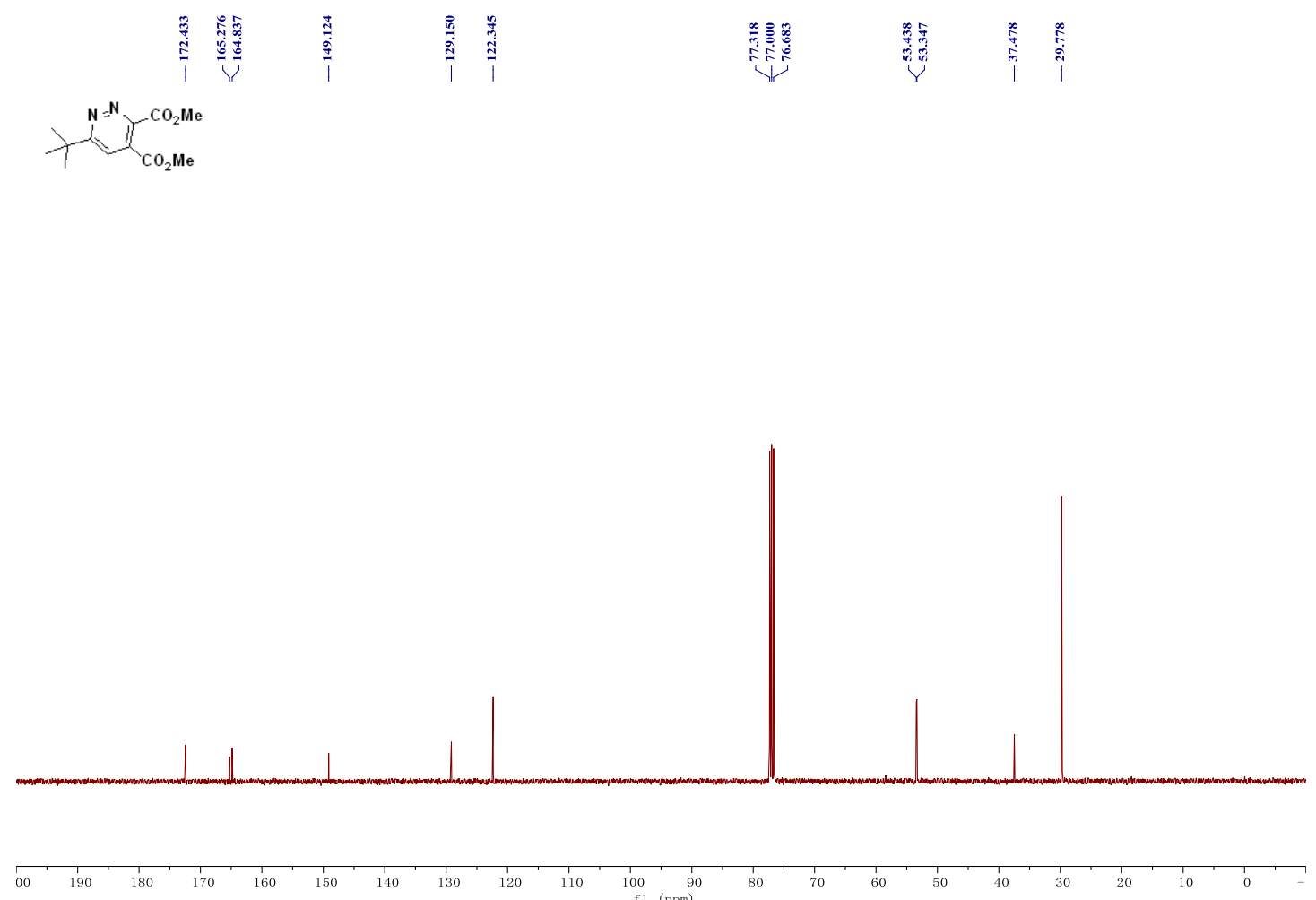

Fig. $\mathbf{S 1 4}{ }^{13} \mathrm{C}$ NMR of compound $2 \mathrm{~g}\left(100 \mathrm{MHz}, \mathrm{CDCl}_{3}\right)$. 


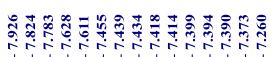

$\underbrace{\mathrm{Ph}}_{\mathrm{CO}_{2} \mathrm{Me}}-\mathrm{CO}_{2} \mathrm{Me}$

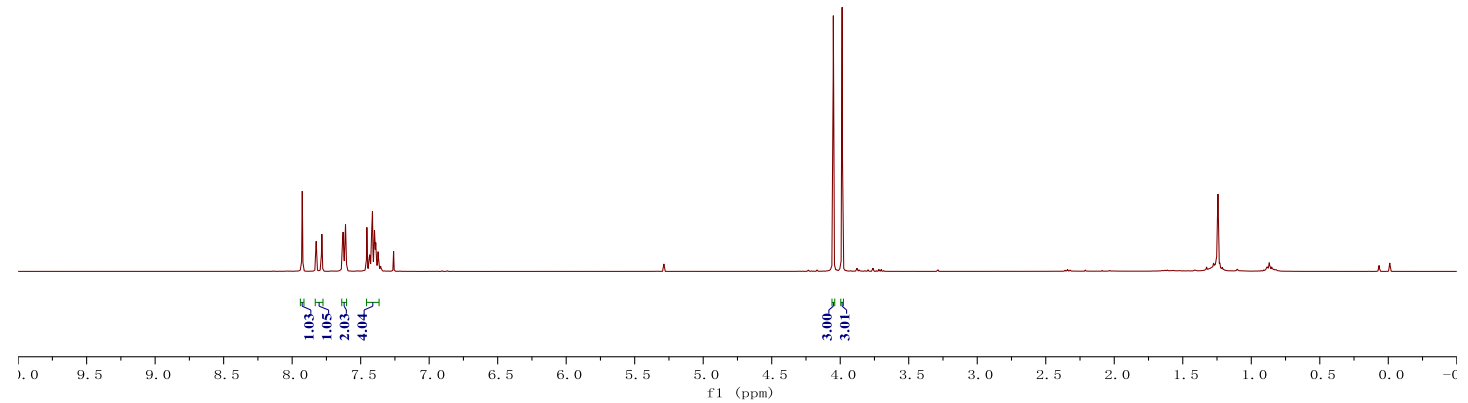

Fig. $\mathbf{S 1 5}{ }^{1} \mathrm{H}$ NMR of compound $\mathbf{2 h}\left(400 \mathrm{MHz}, \mathrm{CDCl}_{3}\right)$.
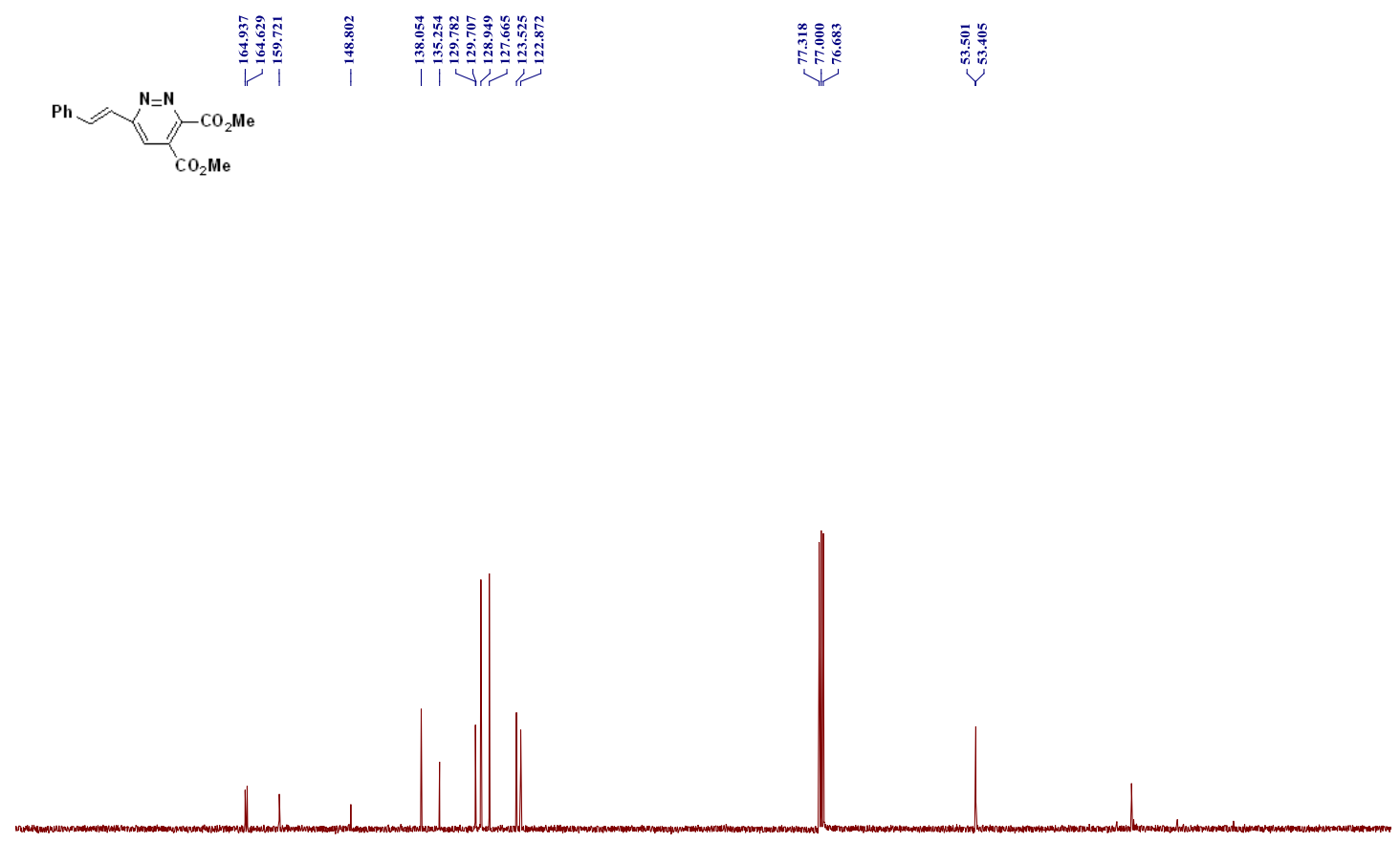

$\begin{array}{lllllllllll}190 & 180 & 170 & 160 & 150 & 140 & 130 & 120 & 110 & 100 & 90 \\ & & & & & & & & & & \end{array}$

Fig. $\mathbf{S 1 6}{ }^{13} \mathrm{C}$ NMR of compound $\mathbf{2 h}\left(100 \mathrm{MHz}, \mathrm{CDCl}_{3}\right)$. 


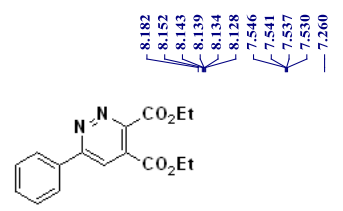

(1) $\mathrm{CO}_{2} \mathrm{E}$

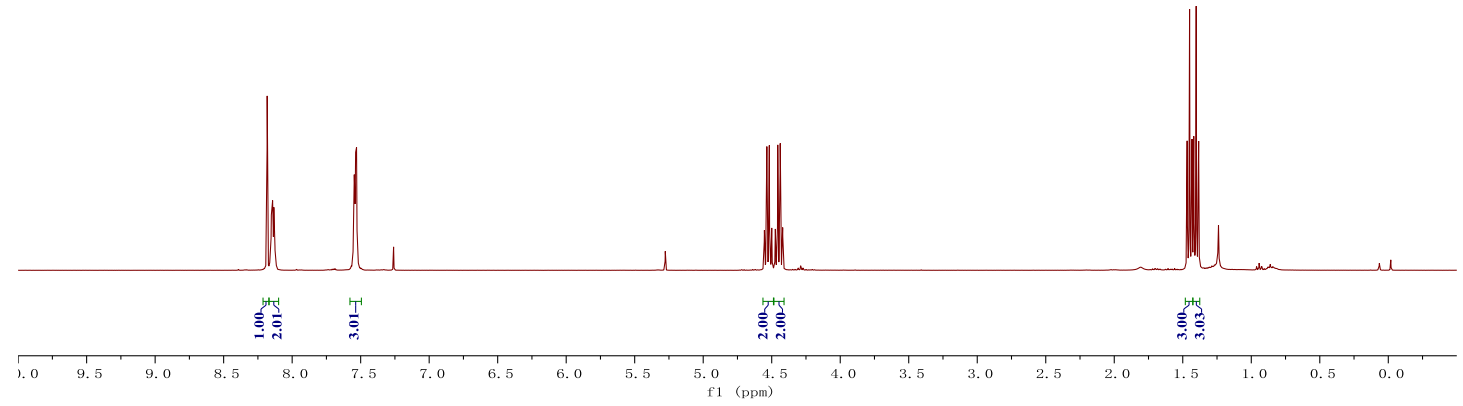

Fig. S17 ${ }^{1} \mathrm{H}$ NMR of compound $2 \mathbf{i}\left(400 \mathrm{MHz}, \mathrm{CDCl}_{3}\right)$.
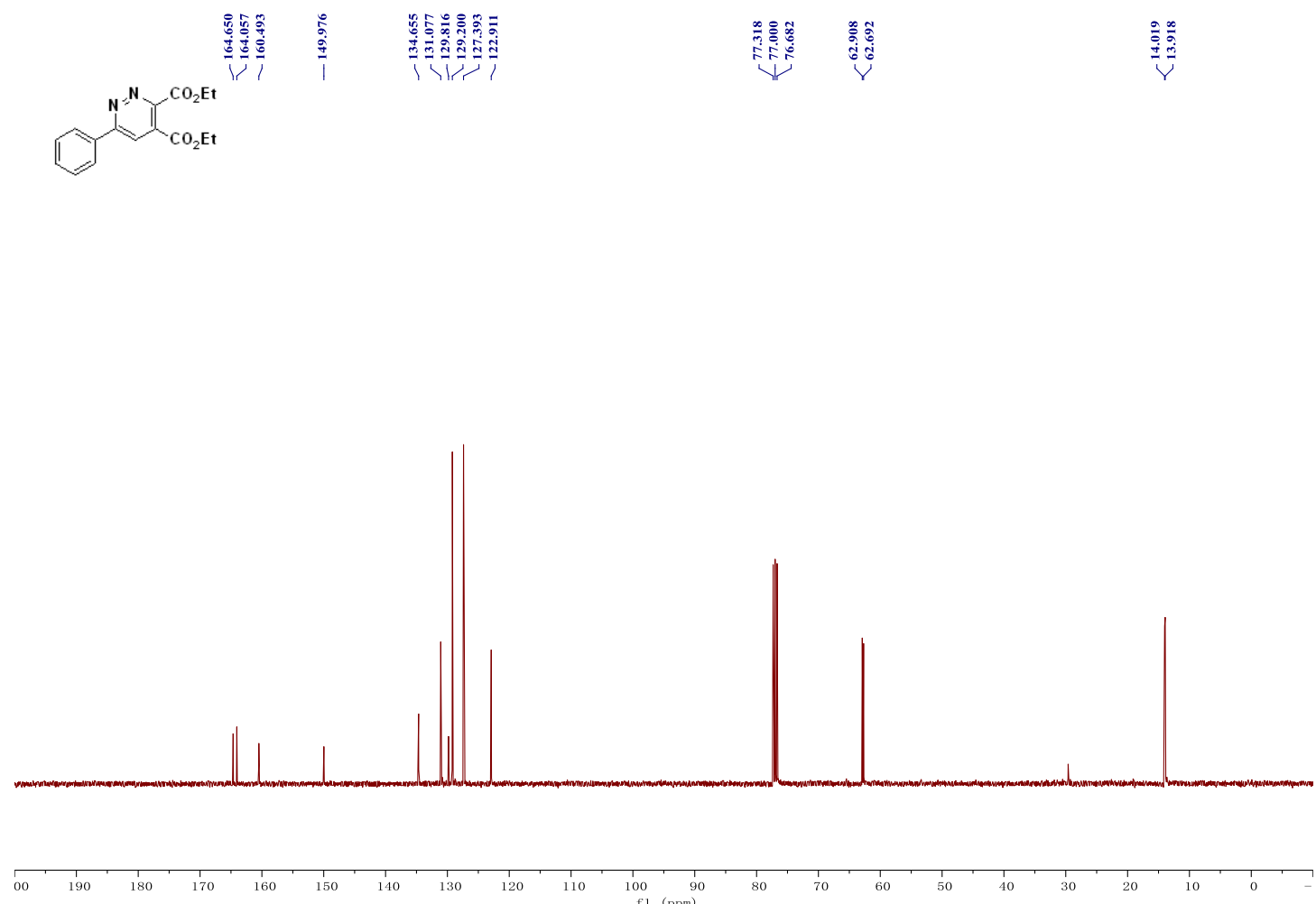

Fig. $\mathbf{S 1 8}{ }^{13} \mathrm{C}$ NMR of compound $2 \mathbf{i}\left(100 \mathrm{MHz}, \mathrm{CDCl}_{3}\right)$. 
$\int_{\mathrm{CO}_{2} \mathrm{Me}}^{\mathrm{HN}-\mathrm{N}} \mathrm{C}-\mathrm{CO}_{2} \mathrm{Me}$

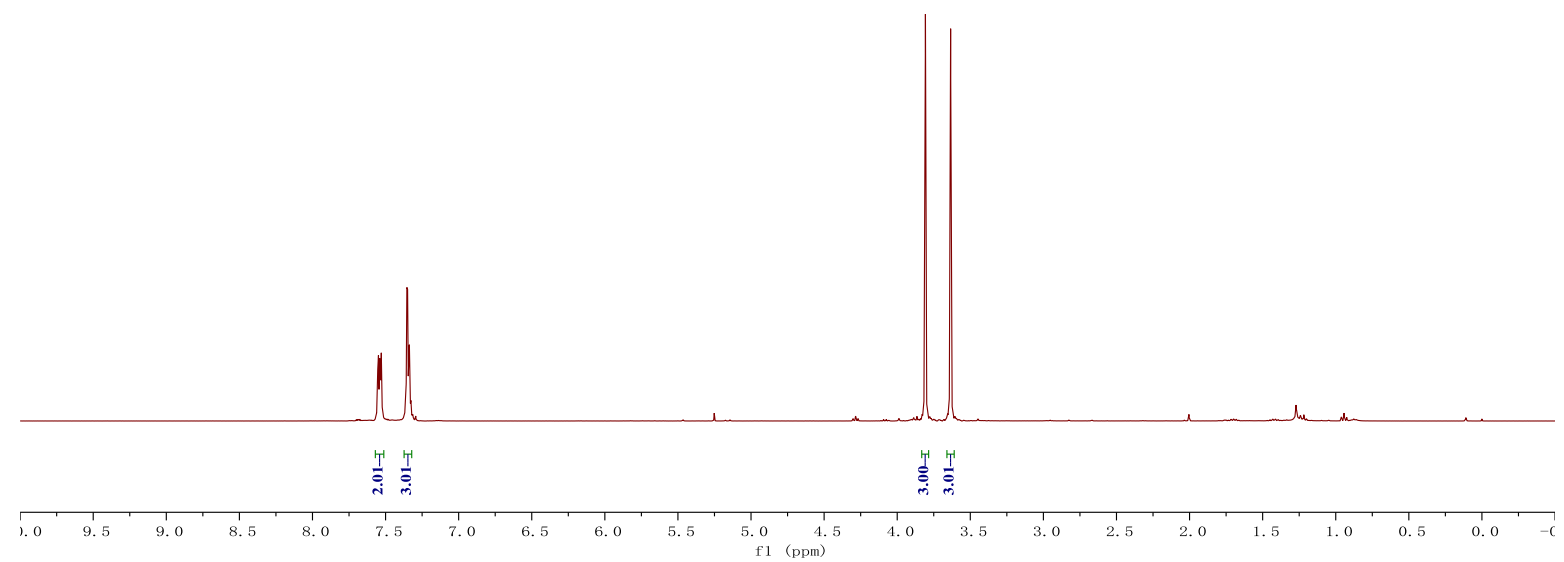

Fig. S19 ${ }^{1} \mathrm{H}$ NMR of compound $\mathbf{3 a}\left(400 \mathrm{MHz}, \mathrm{CDCl}_{3}\right)$.
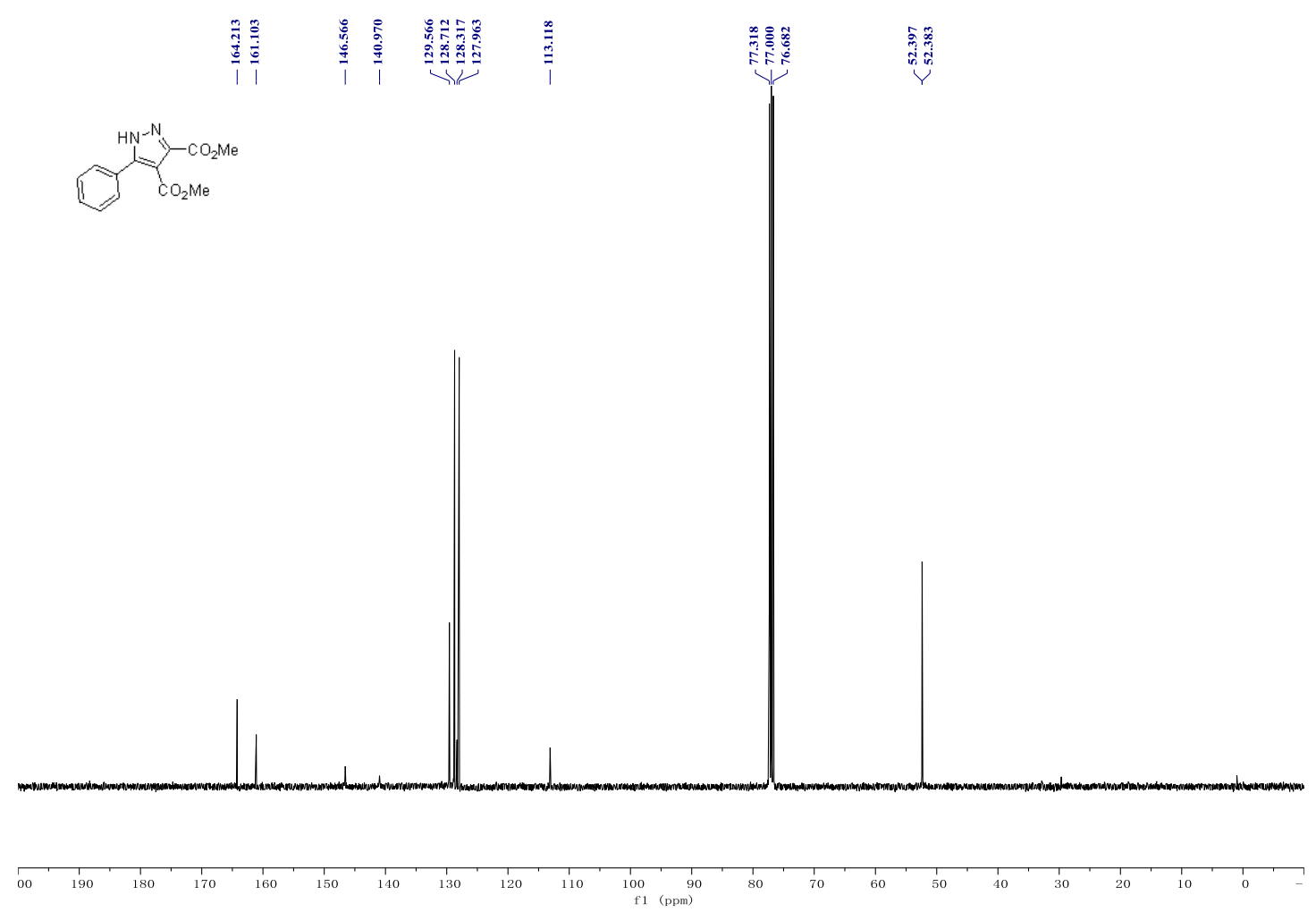

Fig. S20 ${ }^{13} \mathrm{C}$ NMR of compound $\mathbf{3 a}\left(100 \mathrm{MHz}, \mathrm{CDCl}_{3}\right)$. 

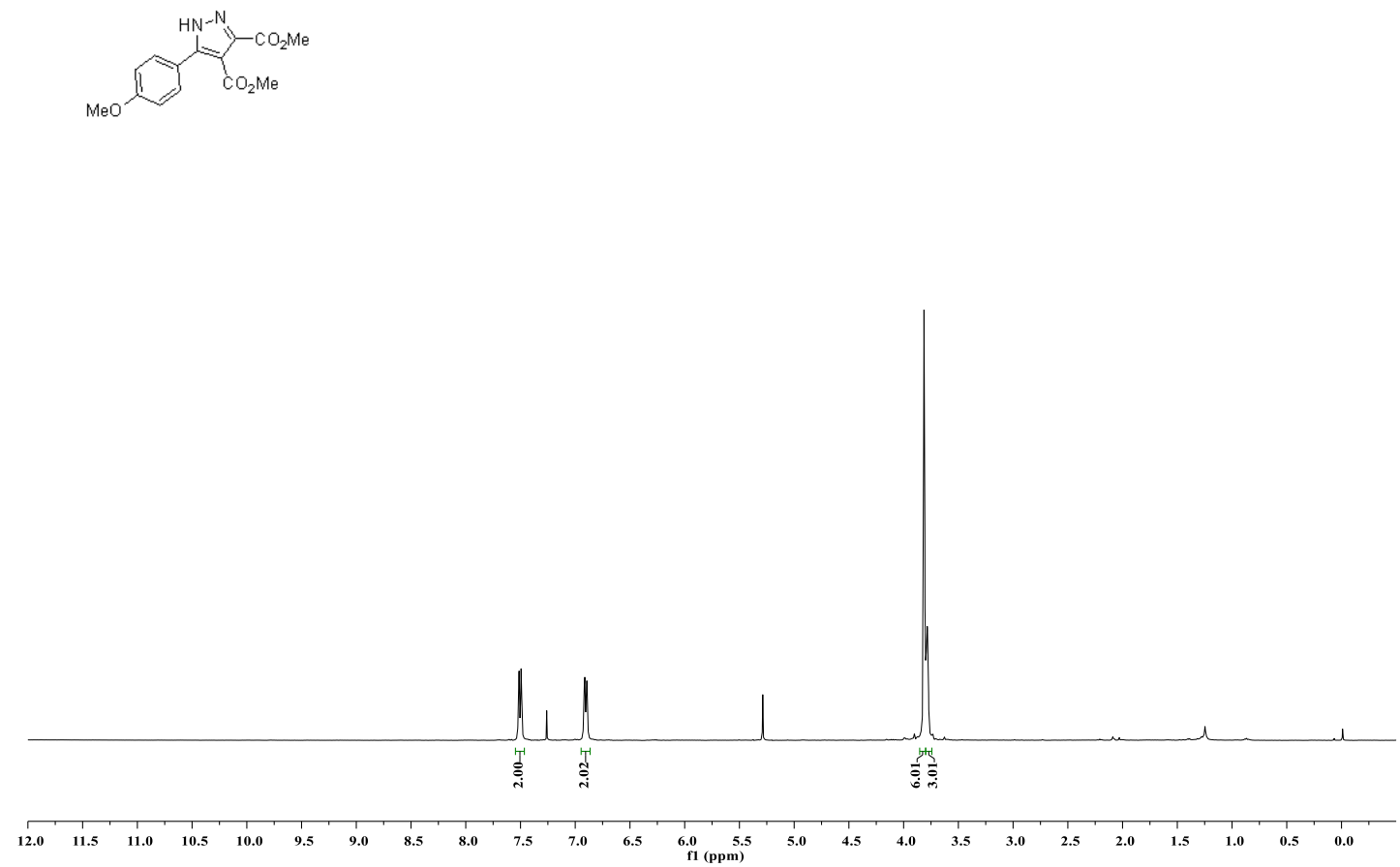

Fig. S21 ${ }^{1} \mathrm{H}$ NMR of compound $\mathbf{3 b}\left(400 \mathrm{MHz}, \mathrm{CDCl}_{3}\right)$.
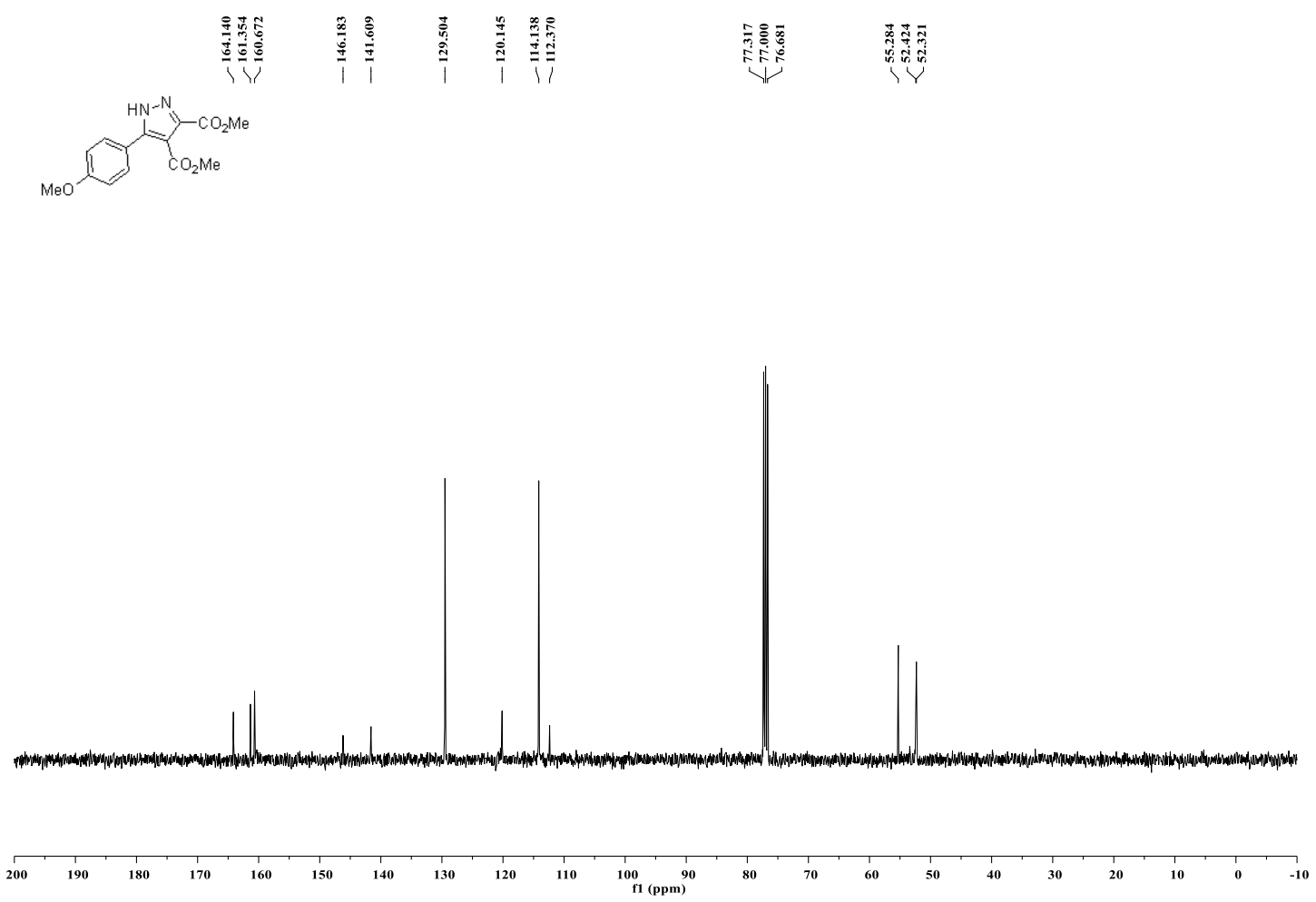

Fig. S22 ${ }^{13} \mathrm{C}$ NMR of compound $\mathbf{3 b}\left(100 \mathrm{MHz}, \mathrm{CDCl}_{3}\right)$. 


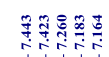
प1ए

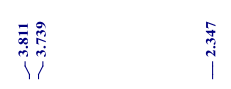
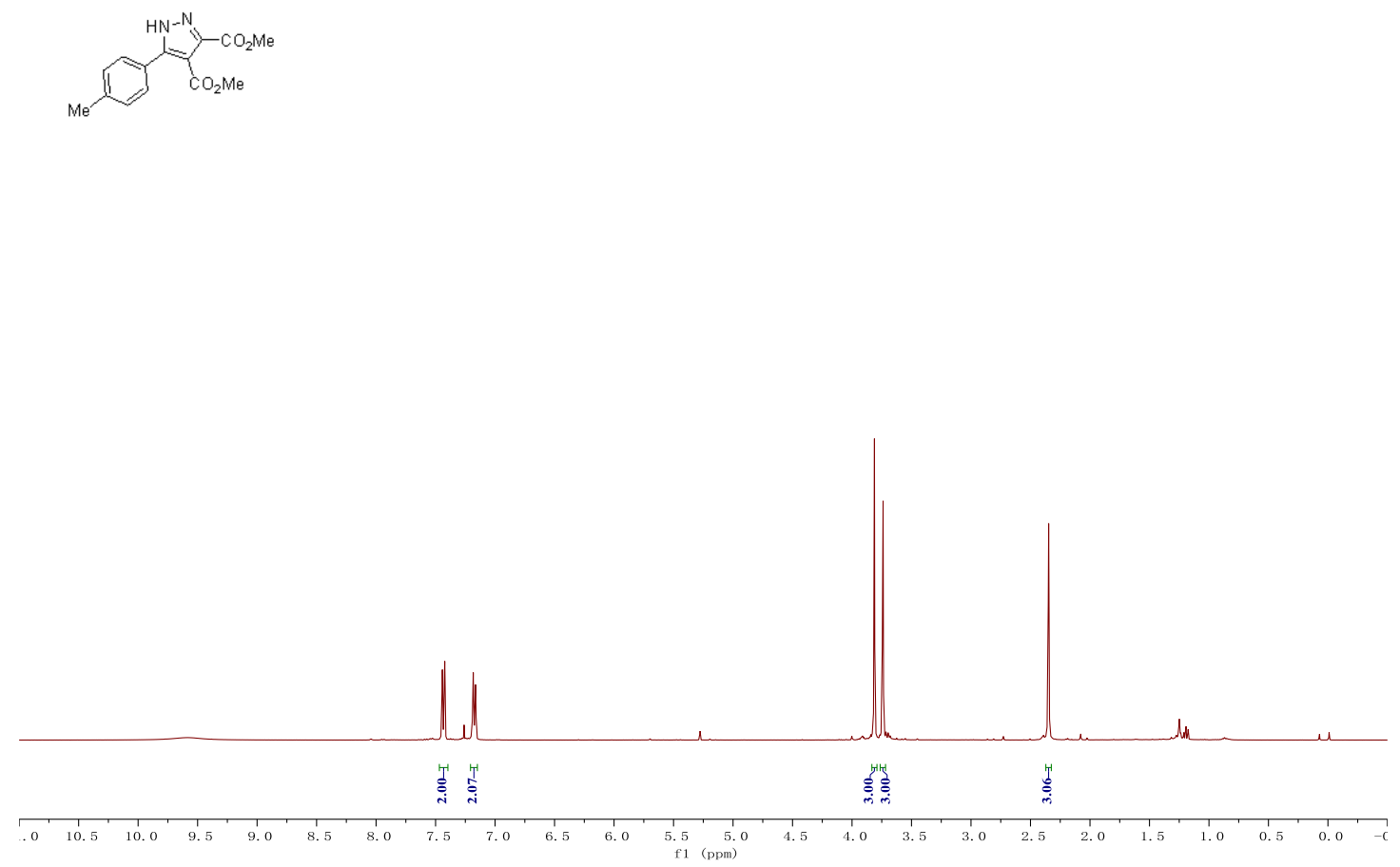

Fig. S23 ${ }^{1} \mathrm{H}$ NMR of compound $\mathbf{3 c}\left(400 \mathrm{MHz}, \mathrm{CDCl}_{3}\right)$.
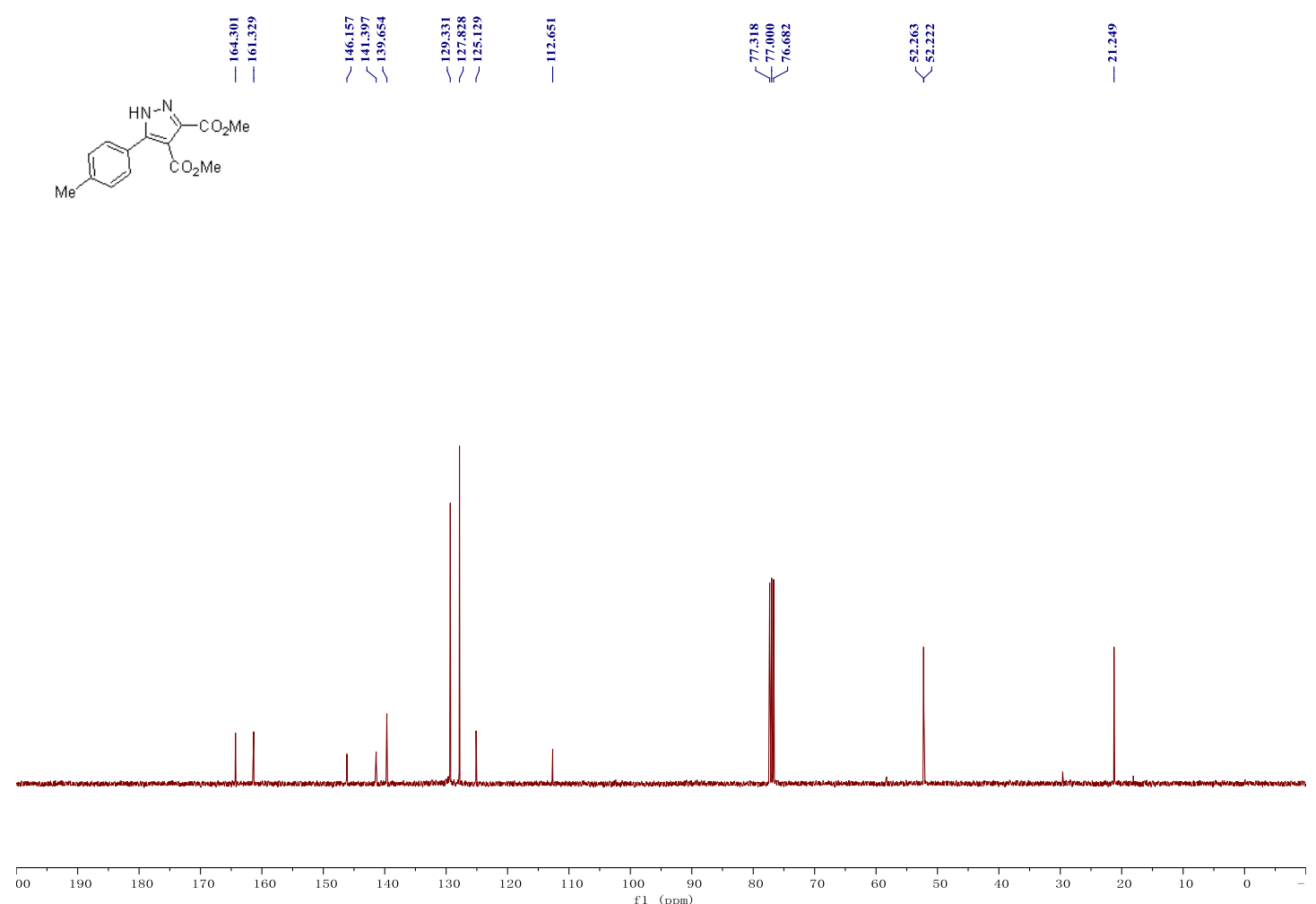

Fig. S24 ${ }^{13} \mathrm{C}$ NMR of compound 3c $\left(100 \mathrm{MHz}, \mathrm{CDCl}_{3}\right)$. 


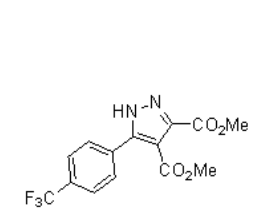

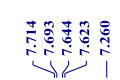

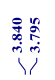

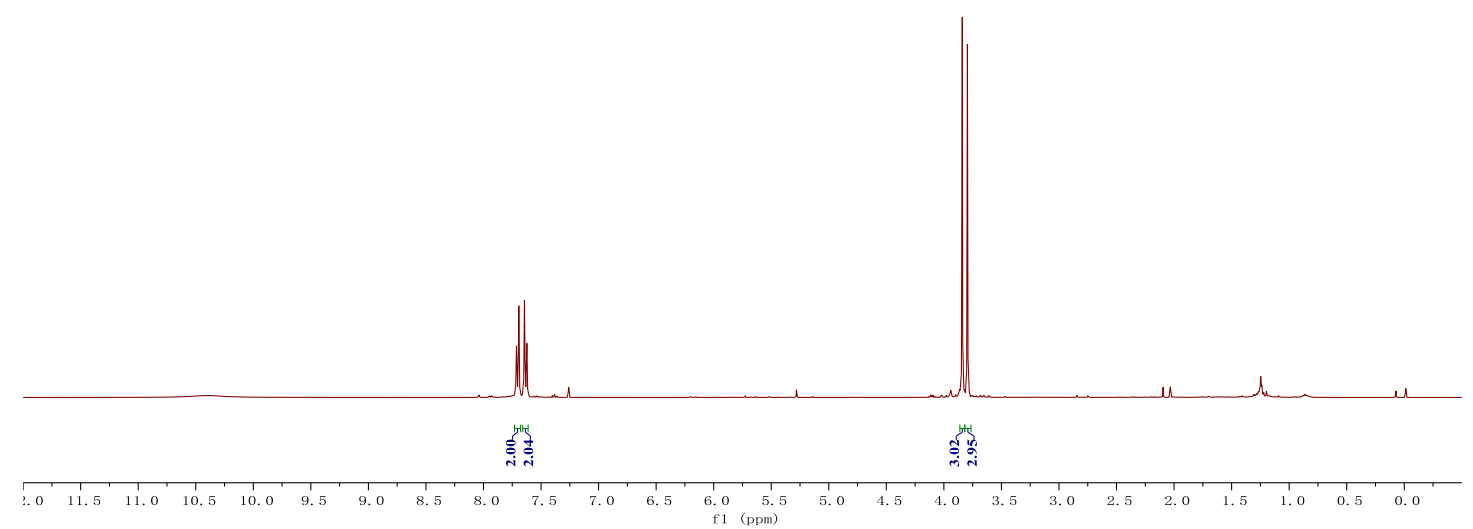

Fig. S25 ${ }^{1} \mathrm{H}$ NMR of compound $\mathbf{3 d}\left(400 \mathrm{MHz}, \mathrm{CDCl}_{3}\right)$.
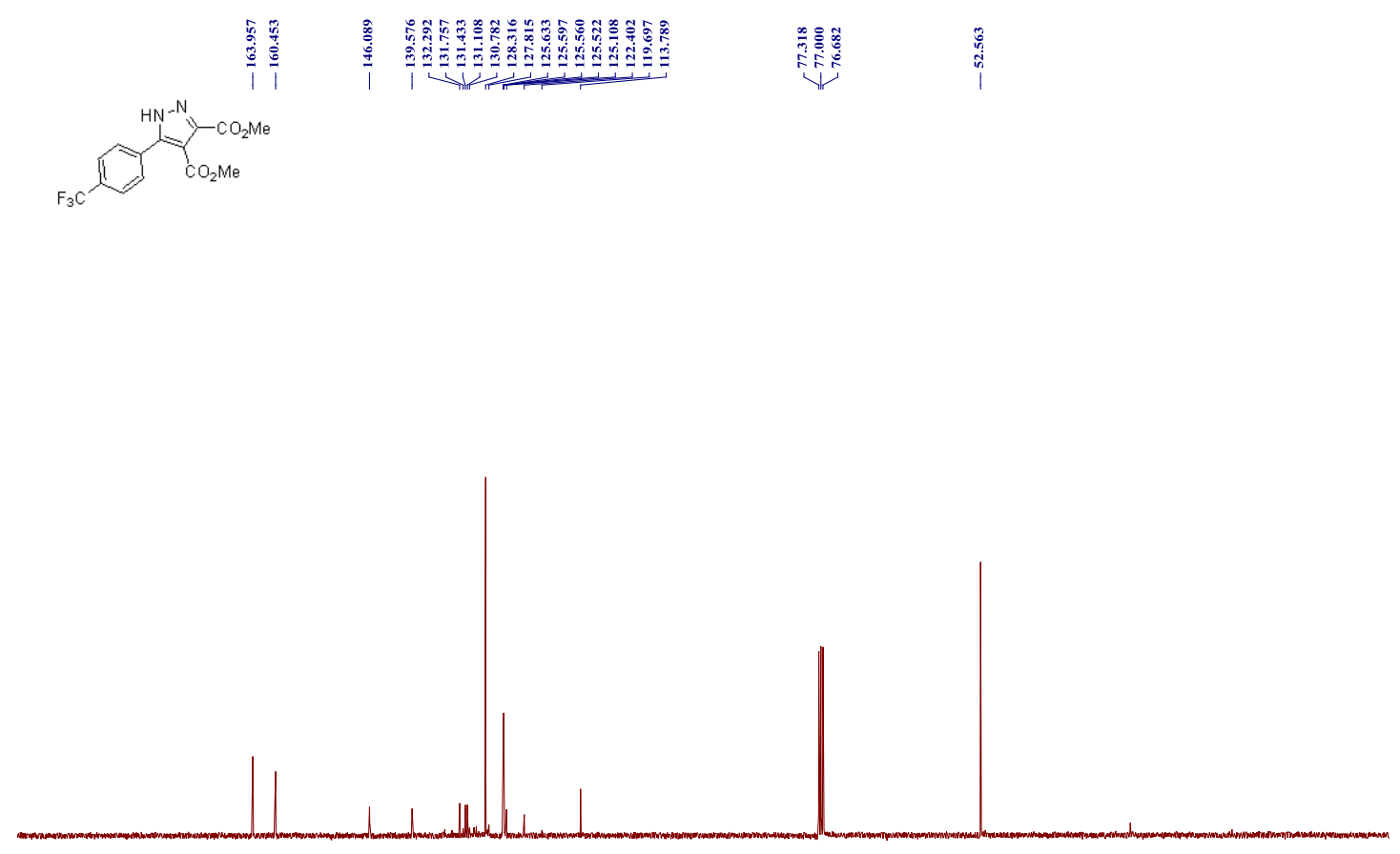

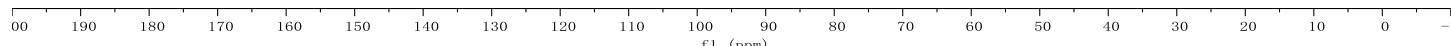

Fig. S26 ${ }^{13} \mathrm{C}$ NMR of compound $\mathbf{3 d}\left(100 \mathrm{MHz}, \mathrm{CDCl}_{3}\right)$. 


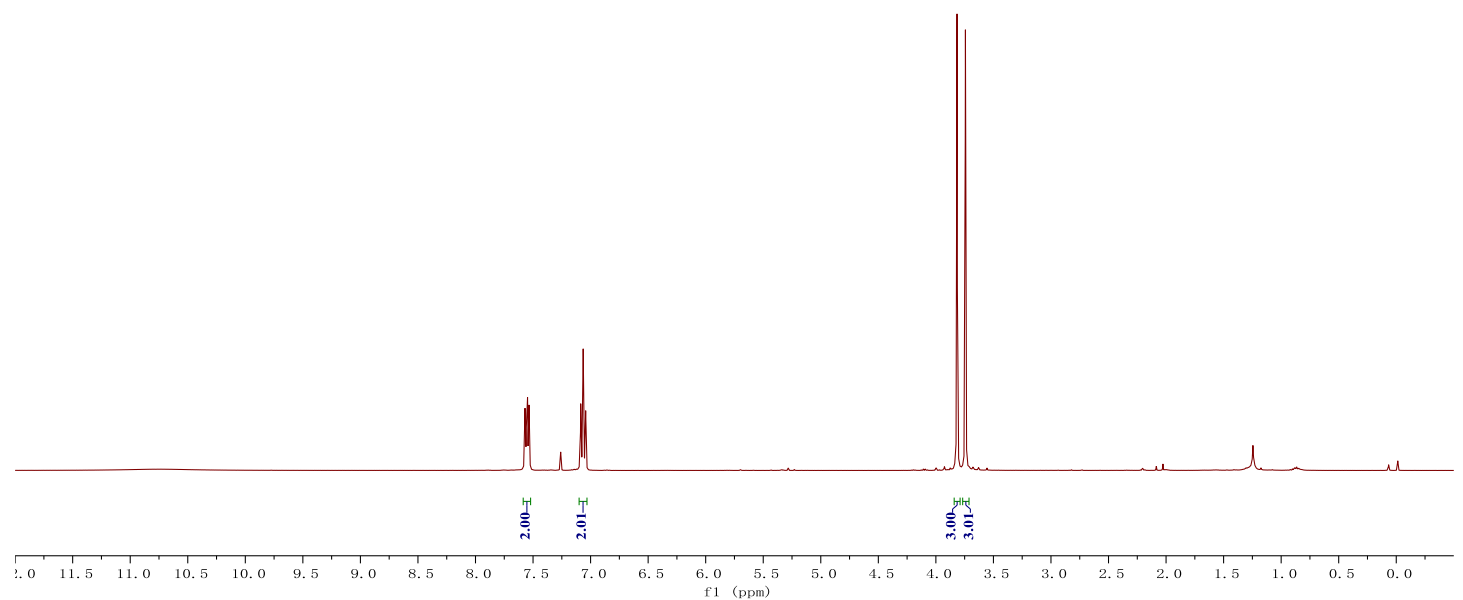

Fig. S27 ${ }^{1} \mathrm{H}$ NMR of compound $\mathbf{3 e}\left(400 \mathrm{MHz}, \mathrm{CDCl}_{3}\right)$.

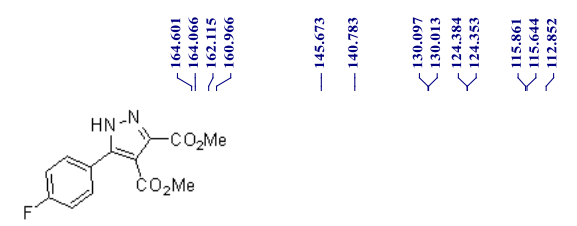

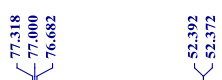
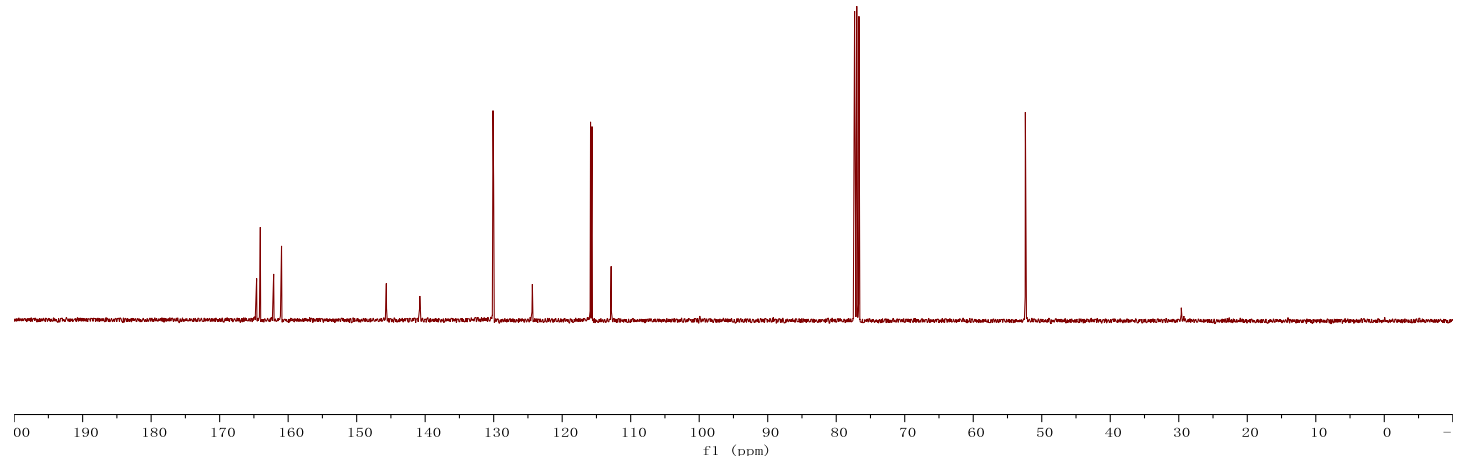

Fig. S28 ${ }^{13} \mathrm{C}$ NMR of compound $\mathbf{3 e}\left(100 \mathrm{MHz}, \mathrm{CDCl}_{3}\right)$. 


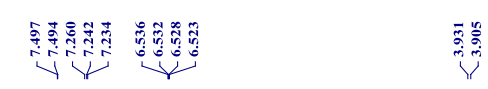

$\overbrace{0}^{\mathrm{HN}-\mathrm{N}} \mathrm{CO}_{2} \mathrm{Me}$

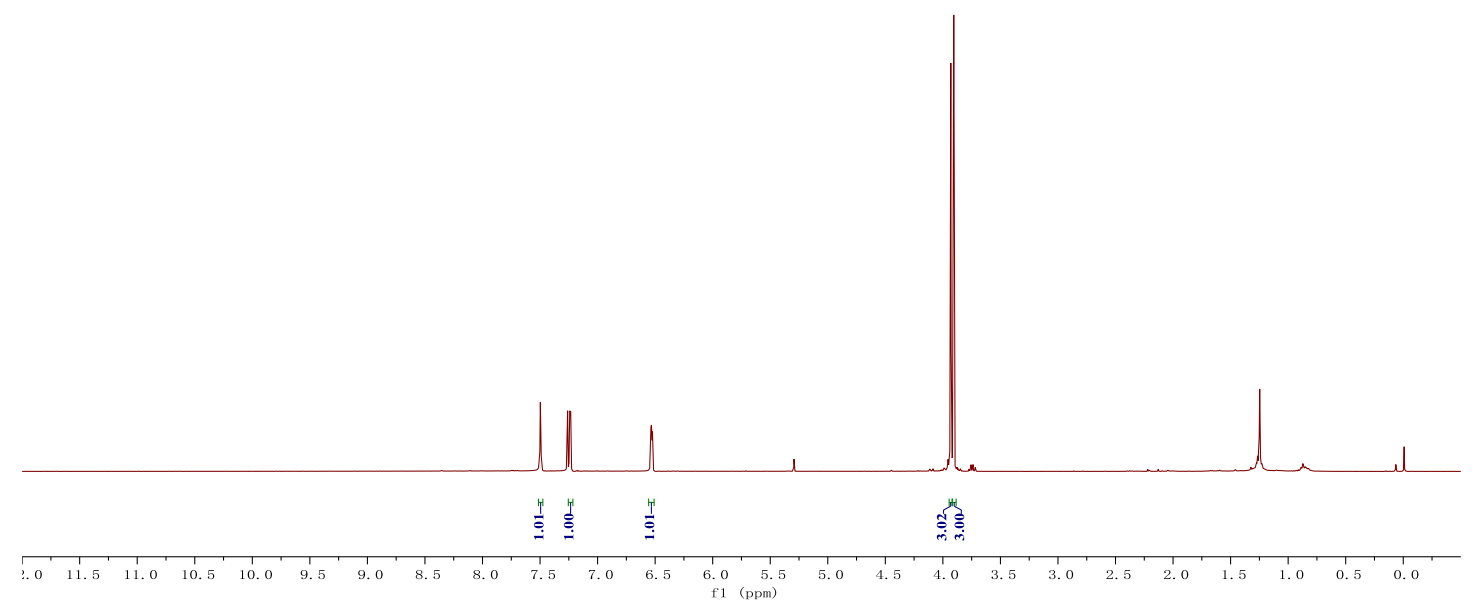

Fig. S29 ${ }^{1} \mathrm{H}$ NMR of compound $\mathbf{3 f}\left(400 \mathrm{MHz}, \mathrm{CDCl}_{3}\right)$.

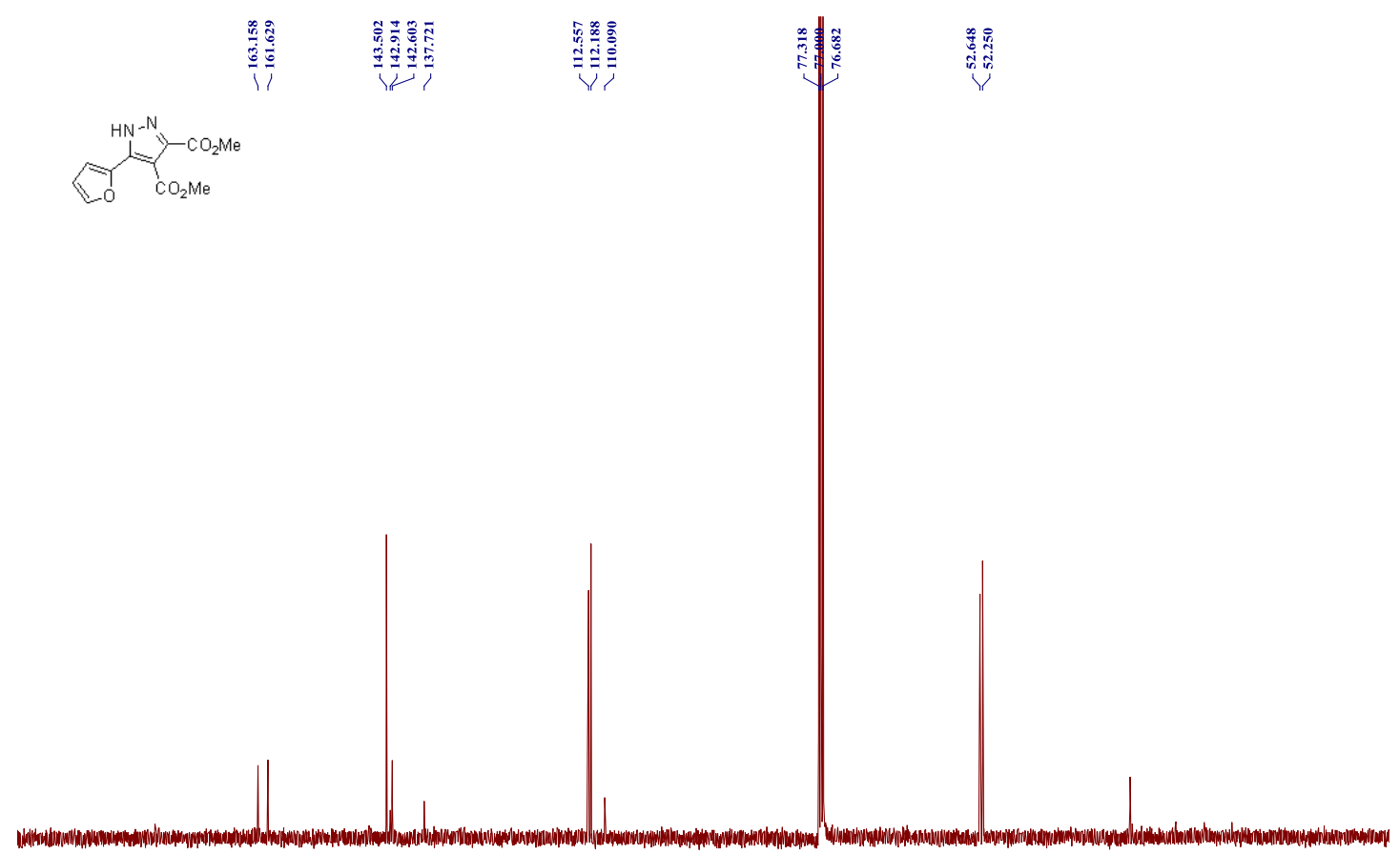

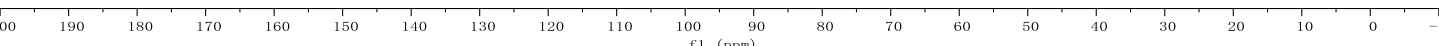

Fig. S30 ${ }^{13} \mathrm{C}$ NMR of compound $3 \mathbf{f}\left(100 \mathrm{MHz}, \mathrm{CDCl}_{3}\right)$. 
$\mathrm{CO}_{2} \mathrm{Me}$

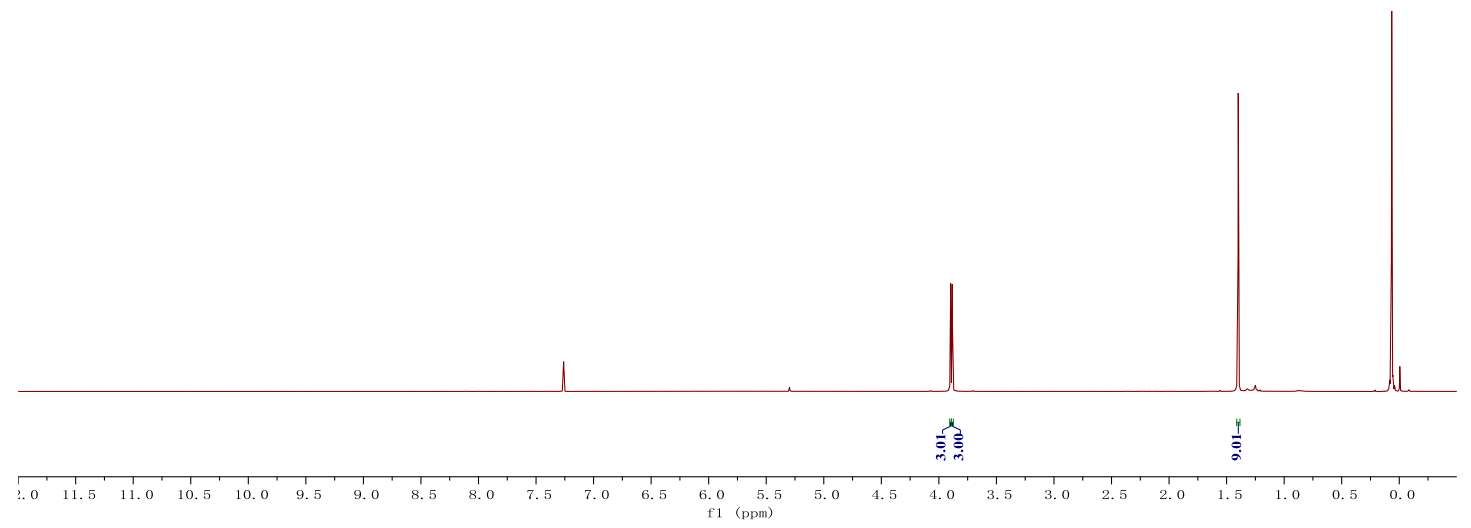

Fig. S31 ${ }^{1} \mathrm{H}$ NMR of compound $\mathbf{3 g}\left(400 \mathrm{MHz}, \mathrm{CDCl}_{3}\right)$.

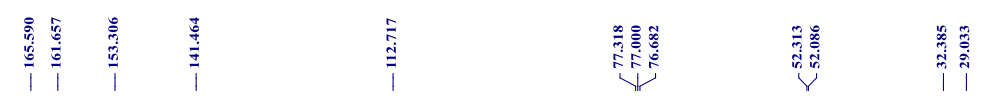

$\mathrm{CO}_{2}^{\mathrm{Me}}$

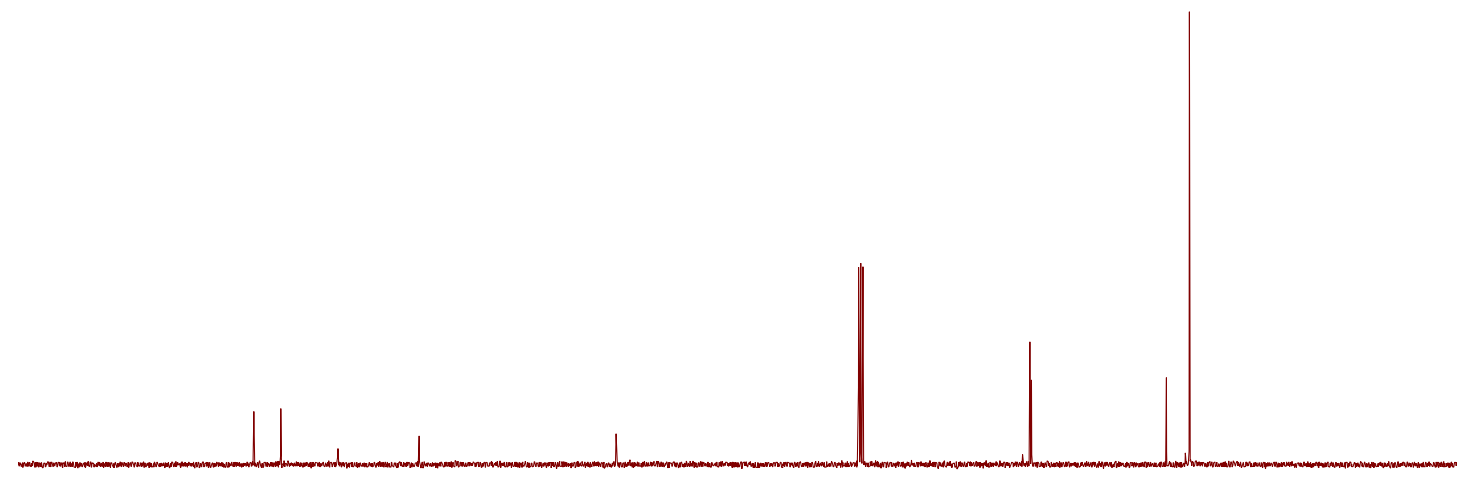

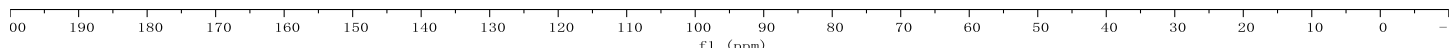

Fig. $\mathbf{S 3 2}{ }^{13} \mathrm{C}$ NMR of compound $\mathbf{3 g}\left(100 \mathrm{MHz}, \mathrm{CDCl}_{3}\right)$. 
$\mathrm{Ph}_{\mathrm{CO}_{2} \mathrm{Me}}^{\mathrm{HN}^{-\mathrm{N}} \curvearrowright \mathrm{CO}_{2} \mathrm{Me}}$

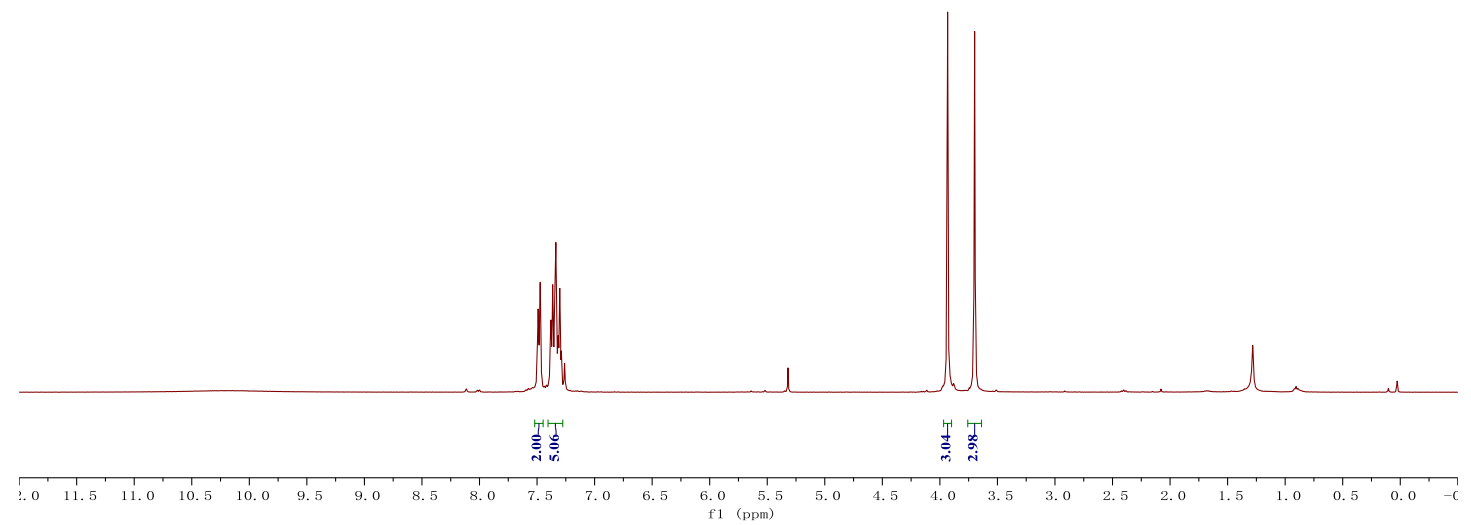

Fig. $\mathbf{S 3 3}{ }^{1} \mathrm{H}$ NMR of compound $\mathbf{3 h}\left(400 \mathrm{MHz}, \mathrm{CDCl}_{3}\right)$.

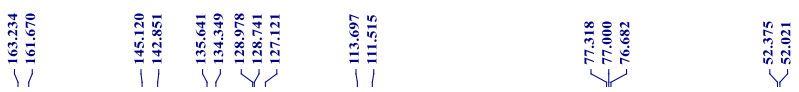

$\underset{\mathrm{CO}_{2} \mathrm{Me}}{\mathrm{CO}_{2} \mathrm{Me}}$

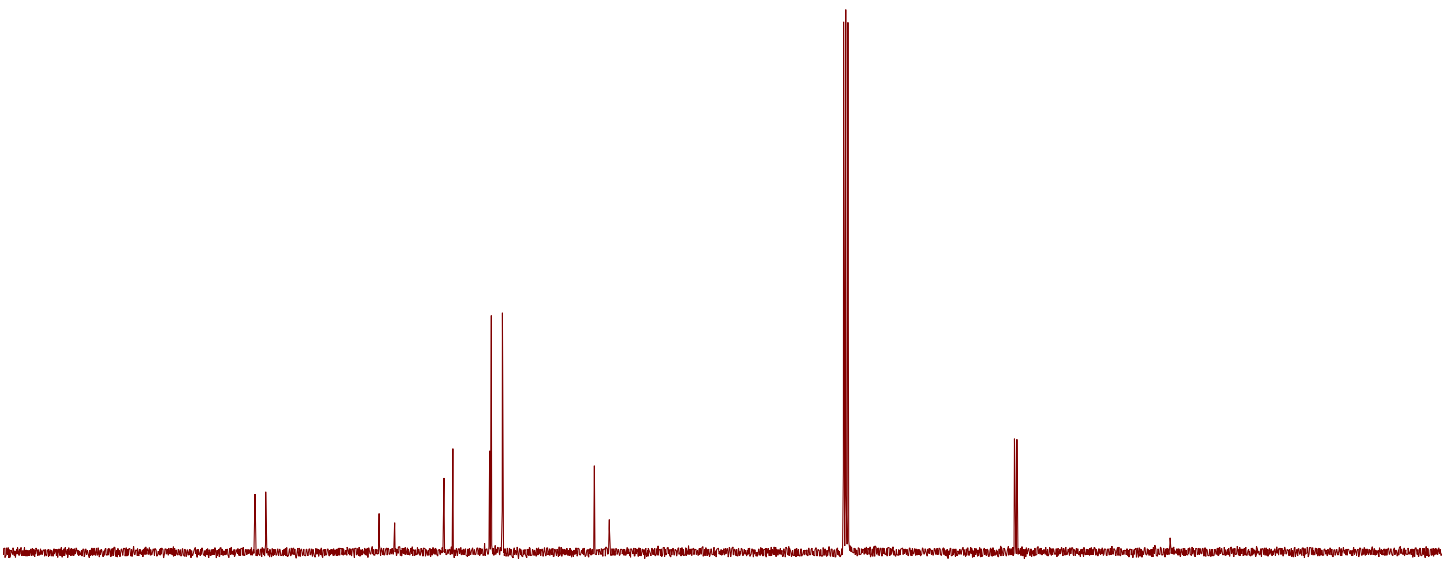

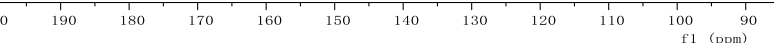

Fig. $\mathbf{S 3 4}{ }^{13} \mathrm{C}$ NMR of compound $\mathbf{3 h}\left(100 \mathrm{MHz}, \mathrm{CDCl}_{3}\right)$. 
$\mathrm{CO}_{2} \mathrm{Et}$

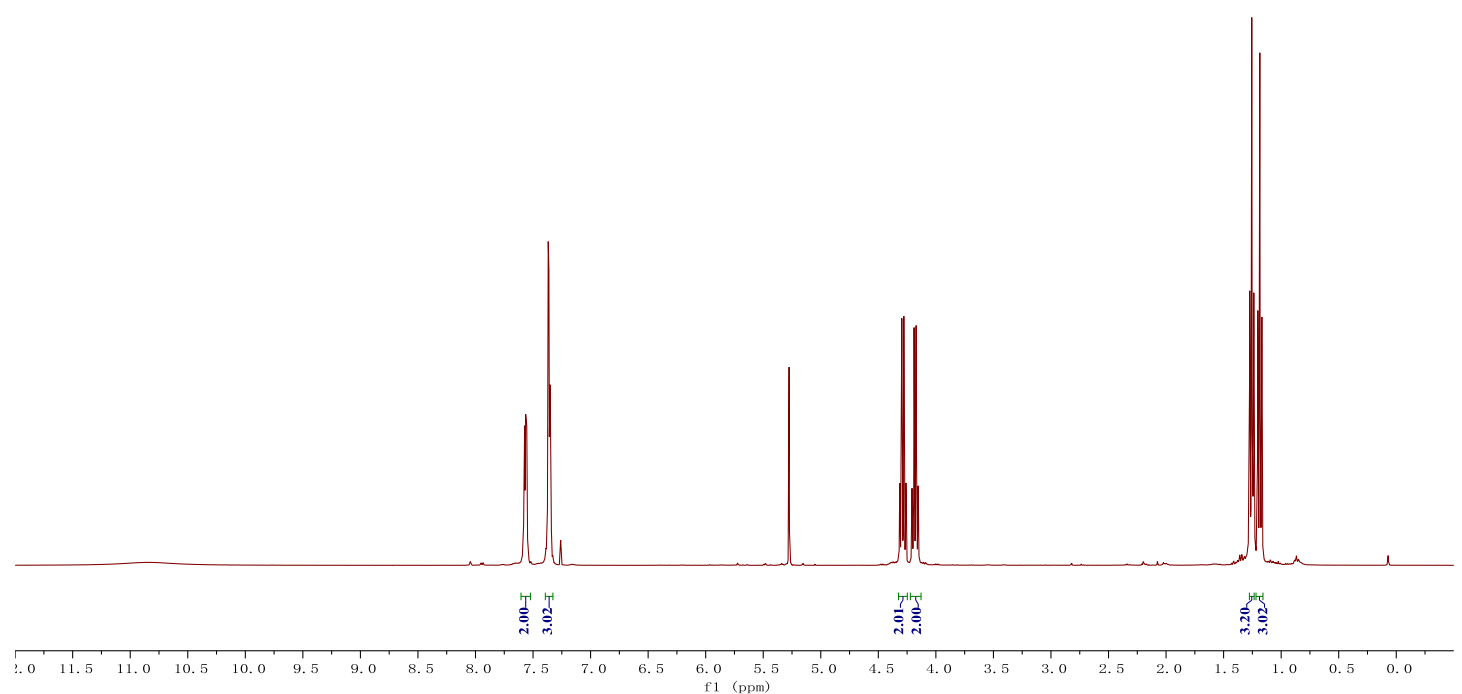

Fig. S35 ${ }^{1} \mathrm{H}$ NMR of compound $\mathbf{3 i}\left(400 \mathrm{MHz}, \mathrm{CDCl}_{3}\right)$.
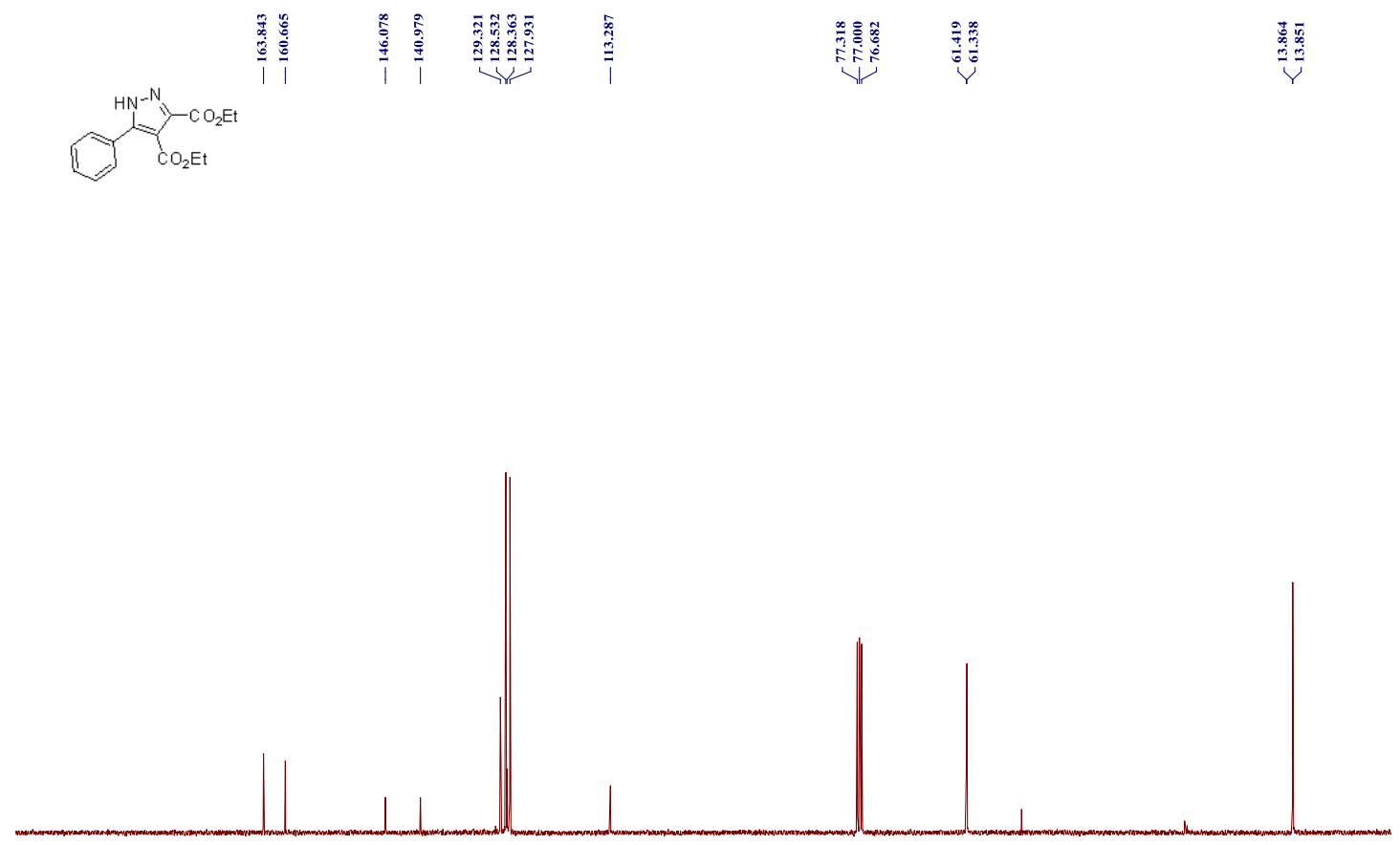

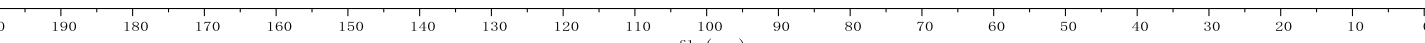

Fig. S36 ${ }^{13} \mathrm{C}$ NMR of compound $3 \mathbf{i}\left(100 \mathrm{MHz}, \mathrm{CDCl}_{3}\right)$. 
2.1 The Crystal Structure Information of Compound 2a

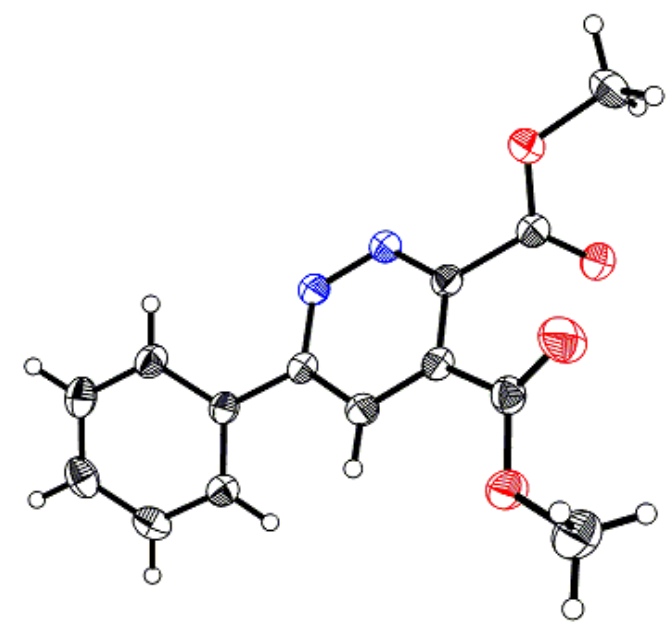

Thermal ellipsoids are drawn at the $30 \%$ probability level 
Table S1 Crystal data and structure refinement for $\mathbf{2 a}$.

Identification code

2a

Empirical formula

$\mathrm{C}_{14} \mathrm{H}_{12} \mathrm{~N}_{2} \mathrm{O}_{4}$

Formula weight

272.26

Temperature/K

296.15

Crystal system

monoclinic

Space group

$\mathrm{P} 2{ }_{1} / \mathrm{c}$

$\mathrm{a} / \AA$

15.261(4)

$\mathrm{b} / \AA$

$7.494(2)$

$\mathrm{c} / \AA$

11.699(3)

$\alpha /{ }^{\circ}$

90.00

$\beta /{ }^{\circ}$

$108.200(4)$

$\gamma /{ }^{\circ}$

90.00

Volume $/ \AA^{3}$

$1271.0(6)$

Z

4

$\rho_{\text {calc }} \mathrm{g} / \mathrm{cm}^{3}$

1.423

$\mu / \mathrm{mm}^{-1}$

0.106

$\mathrm{F}(000)$

568.0

Crystal size $/ \mathrm{mm}^{3}$

$0.18 \times 0.14 \times 0.12$

Radiation

$\operatorname{MoK} \alpha(\lambda=0.71073)$

$2 \Theta$ range for data collection $/{ }^{\circ} 2.8$ to 52.04

Index ranges

$-14 \leq \mathrm{h} \leq 18,-7 \leq \mathrm{k} \leq 9,-14 \leq 1 \leq 14$

Reflections collected

6721

Independent reflections

$2498\left[R_{\text {int }}=0.0223, R_{\text {sigma }}=0.0269\right]$

Data/restraints/parameters

$2498 / 0 / 183$

Goodness-of-fit on $\mathrm{F}^{2}$

1.046

Final $\mathrm{R}$ indexes $[\mathrm{I}>=2 \sigma(\mathrm{I})] \quad \mathrm{R}_{1}=0.0387, \mathrm{wR}_{2}=0.1025$

Final $\mathrm{R}$ indexes [all data] $\quad \mathrm{R}_{1}=0.0497, \mathrm{wR}_{2}=0.1117$

Largest diff. peak/hole / e $\AA^{-3} 0.15 /-0.18$ 
2.2 The Crystal Structure Information of Compound 3g

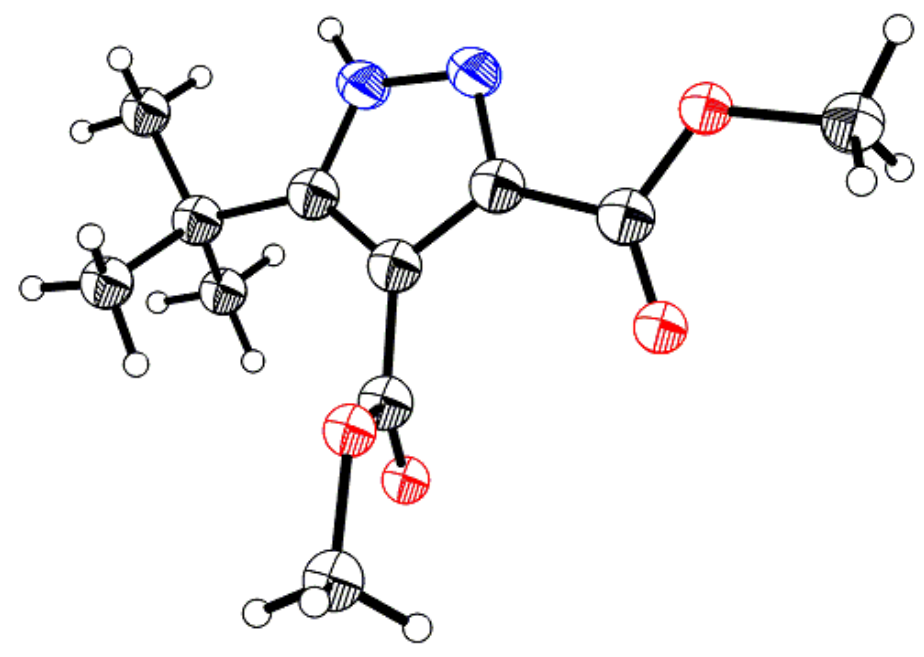

Thermal ellipsoids are drawn at the $30 \%$ probability level 
Table S2 Crystal data and structure refinement fo $\mathbf{3 g}$.

Identification code

$3 g$

Empirical formula

$\mathrm{C}_{44} \mathrm{H}_{64} \mathrm{~N}_{8} \mathrm{O}_{16}$

Formula weight

961.03

Temperature/K

293.65(10)

Crystal system

triclinic

Space group

$\mathrm{P}-1$

$\mathrm{a} / \AA$

$13.8348(8)$

$\mathrm{b} / \AA$

$14.2875(8)$

$\mathrm{c} / \AA$

$15.0081(10)$

$\alpha /{ }^{\circ}$

$75.879(6)$

$\beta /{ }^{\circ}$

$87.440(5)$

$\gamma /{ }^{\circ}$

65.866(6)

Volume $/ \AA^{3}$

2620.4(3)

$\mathrm{Z}$

$\rho_{\text {calc }} / \mathrm{cm}^{3}$

2

1.218

$\mu / \mathrm{mm}^{-1}$

0.093

$\mathrm{F}(000)$

1024.0

Crystal size $/ \mathrm{mm}^{3}$

$0.17 \times 0.14 \times 0.12$

Radiation

$\operatorname{MoK} \alpha(\lambda=0.71073)$

$2 \Theta$ range for data collection $/{ }^{\circ} 6.722$ to 52.044

Index ranges

$-17 \leq \mathrm{h} \leq 16,-17 \leq \mathrm{k} \leq 16,-18 \leq 1 \leq 10$

Reflections collected

19065

Independent reflections

$10286\left[R_{\text {int }}=0.0491, R_{\text {sigma }}=0.0906\right]$

Data/restraints/parameters

$10286 / 0 / 645$

Goodness-of-fit on $\mathrm{F}^{2}$

1.036

Final $R$ indexes $[\mathrm{I}>=2 \sigma(\mathrm{I})] \quad \mathrm{R}_{1}=0.0815, \mathrm{wR}_{2}=0.1875$

Final $\mathrm{R}$ indexes [all data] $\quad \mathrm{R}_{1}=0.1475, \mathrm{wR}_{2}=0.2496$

Largest diff. peak/hole / e $\AA^{-3} 0.31 /-0.37$ 